\title{
Glucocorticoids promote breast cancer metastasis
}

\section{Obradovic, Milan M. S.}

2019-03-28

Obradovic, M M S , Hamelin , B , Manevski , N , Couto , J P , Sethi , A, Coissieux , M-M , Munst , S , Okamoto , R , Kohler , H , Schmidt , A \& Bentires-Alj , M 2019 , ' Glucocorticoids promote breast cancer metastasis ' , Nature , vol. 567 , no. 7749 , pp. 540-+ . https://doi.org/10.1038/s41586-019-10

http://hdl.handle.net/10138/327527

https://doi.org/10.1038/s41586-019-1019-4

unspecified

acceptedVersion

Downloaded from Helda, University of Helsinki institutional repository.

This is an electronic reprint of the original article.

This reprint may differ from the original in pagination and typographic detail.

Please cite the original version. 
1 Stress hormones induce breast intra-tumour heterogeneity and metastases

2 Milan Obradović ${ }^{1,2}$, Baptiste Hamelin ${ }^{1}$, Nenad Manevski ${ }^{3}$, Joana Pinto do Couto ${ }^{1,2}$, Simone Münst $^{4}$, Ryoko

3 Okamoto $^{1,2}$, Sandrine Bichet ${ }^{2}$, Hubertus Kohler ${ }^{2}$, Hans-Rudolf Hotz $^{2}$, Alexander Schmidt ${ }^{5}$, Mohamed

4 Bentires-Alj ${ }^{1,2 *}$

$5 \quad{ }^{1}$ Department of Biomedicine, Department of Surgery, University Hospital Basel, University of Basel, Switzerland

$6 \quad{ }^{2}$ Friedrich Miescher Institute for Biomedical Research, Basel, Switzerland

$7 \quad{ }^{3}$ UCB Pharma, DMPK, 216 Bath Road, SL1 3WE, Slough, UK

$8 \quad{ }^{4}$ Institute of Pathology, University Hospital Basel, University of Basel, Switzerland

$9 \quad{ }^{5}$ Proteomics Core Facility, Biozentrum, University of Basel, Basel, Switzerland

10 *correspondence to: Mohamed Bentires-Alj, Department of Biomedicine, Department of Surgery, University Hospital

11 Basel, University of Basel, Switzerland M.Bentires-Alj@unibas.ch

12 Diversity within or between a tumour and metastases, known as intra-patient tumour heterogeneity 13 develops during disease progression, and is a serious hurdle for therapy ${ }^{1,2,3}$. Metastasis is the fatal 14 hallmark of cancer and mechanisms of colonization, the most complex step of the metastatic 15 cascade $^{4,5}$, remain ill-defined. Better understanding of cellular and molecular processes underlying intra-patient tumour heterogeneity and metastasis are pivotal for the success of personalized cancer

17 treatment. Here, transcriptional profiling of tumours and matched metastases showed cancer sitespecific phenotypes, and identified increased glucocorticoid receptor (GR) activity in the distant metastases. GR has been shown to mediate the effects of stress hormones and of their synthetic derivatives, widely used in the clinic as anti-inflammatory and immunosuppressive. We show that increase in stress hormones during breast cancer progression resulted in GR activation in the distant metastatic sites, increased colonization, and ultimately reduced survival. Transcriptomics, proteomics and phosphoproteomics studies revealed that GR activates multiple processes implicated in metastases and increased expression of the kinase ROR1 which correlates with shorter overall survival in patients. Ablation of ROR1 reduced metastatic outgrowth and prolonged survival in preclinical models. Our results suggest that GR activation increases heterogeneity and metastasis. Because glucocorticoids have been widely used in treatment of cancer-related complications, our results call for caution when including such agents in the treatment of breast cancer patients. 
During malignant progression, cancer cells and the patients undergo series of genetic, epigenetic as well as hormonal and immunological changes, which result in a yet insufficiently understood intra-patient tumour heterogeneity ${ }^{1,2,4-8}$. Phenotypic changes of cancer cells are consequence of selection and adaptation mechanisms that enable cancer growth at distant sites such as lungs, liver, bone and brain years after primary tumour diagnosis and removal ${ }^{9,10}$. Intra-patient tumour heterogeneity stems treatment obstacles and spawns discordance in diagnostic markers between primary tumours and matched metastases and may lead to inadequate cancer treatment ${ }^{11,12}$. Yet, our understanding of global phenotypic changes which are established in the distant metastatic sites is still fragmentary ${ }^{13}$. To explore the heterogeneity between tumours and distant metastases in clinically relevant models, we implanted 17 primary derived xenografts (PDX) and cell lines in the mammary gland of NOD-scid IL2rynull (NSG) immunodeficient mice, resected the primary tumour (tumour) and monitored metastatic development. Metastases were found in the lungs, liver, spleen, ovaries and as circulating tumour cells (CTC) (Fig. 1a, Supplementary Table 1, Extended Data Fig. 1a-e). To characterize matched tumours and metastases, we isolated cancer cells based on expression of GFP in MDA-MB 231 model or using the human specific marker CD298 ${ }^{14}$ in case of PDX models, by fluorescence-activated cell sorting (FACS), and performed global transcriptional profiling. Principal component analysis (PCA) revealed that cancer cells mainly cluster based on the site of metastases (Figure 1b, Extended Data Fig. 1f). The most frequently differentially regulated cellular processes between tumours and matched metastases are metabolism, hypoxia, and mTOR signalling (Supplementary Table 2). Next, we used an integrated system for motif activity response analysis (ISMARA) ${ }^{15}$ and we modelled transcription factor activity in these samples and in publicly available datasets ${ }^{16}$. We found a recurrent increase in glucocorticoid receptor activity in metastases (Extended Data Figure 2a-c). Ingenuity Pathway Analysis (IPA) indicated that differential expression between tumours and matched metastases corresponded to the expression profiles evoked by glucocorticoids such as dexamethasone (DEX) and triamcinolone acetonide (Fig. 1d, Extended Data Fig. 1g-i). Next, we measured stress hormone levels in these animals. Cortisol and corticosterone, were increased in the plasma of animals with metastases compared to controls or animals with tumours and no metastases (Fig. 1e, f). Cancer cells isolated from tumours and metastases lacked expression of genes involved in biosynthesis of stress hormones (Extended Data Fig. 2d-g) suggesting that these hormones were not generated by the cancer cells. The levels of the adrenocorticotropic hormone $(\mathrm{ACTH})$, which increases the production and release of cortisol and corticosterone, also increased in the plasma of animals with metastases (Fig. 1g). Taken together, our data reveal enhanced GR activity in breast cancer metastases most likely due to increased levels of glucocorticoids during breast cancer progression.

Breast cancer patients with metastases have increased levels of stress hormones compared to the age matched healthy women or patients without metastases ${ }^{17}$, while abnormal or flattened cortisol rhythms 
were associated with shorter survival in patients with advanced breast cancer ${ }^{18}$. We sought to explore the cell autonomous effect of glucocorticoids in metastasis. To assess the effect of prolonged GR activation, we exposed in vitro breast cancer cells, expressing a control short hairpin RNA (shCTRL) or shRNAs targeting GR (shGR1, shGR2), to DEX for seven consecutive days (Figure 2a, Extended Data Fig. 3a). We found an increased expression of GR $\operatorname{targets}^{7,19,20}$ (FN1, KLF9, ANKRD1, MT2A, VIM, SNAI2, POU5F1, ID3) and in DEX-treated control cells compared to ShGR1 and 2 or untreated cells (Fig. 2 b, Extended Data Fig. 3b-d) and furthermore shGR1 and shGR2 cells failed to express GR activation markers (Extended Data Fig. 3b). Prolonged GR activation is a reversible process as upon withdrawal of DEX, the cells expressed initial levels of GR targets (Extended Data Fig. 3c). Of note, analysis of published breast cancer datasets $^{21,22}$, showed co-occurring expression of GR and its targets (Extended Data Fig. 3d), which correlates with the claudin-low intrinsic breast cancer subtype (Extended Data Fig. 3e).

To address how prolonged GR activation affects lung colonization, we used the experimental metastases assay in which cancer cells are injected i.v.. We inoculated control or GR activated MDA-MB 231 and 4T1 metastatic mammary cancer cells in the lungs of immunodeficient or immunocompetent mice respectively, and found increased metastases in animals injected with DEX-induced cells (Fig. 2c, d). The increase of colonization was not observed in cells lacking GR (Extended Data Fig. 3f) and we did not observe differences in tumour size between shGR and control cells (Extended Data Fig. 3g). Ex vivo activation of GR resulted in no increase in tumour volume when the cells were injected orthotopically (Extended Data Fig. 3h).

Because the experimental metastases assay recapitulates only the last steps of the metastatic cascade $^{23}$, we sought to address the effect of glucocorticoids upon orthotopic transplantation of cancer cells and tumour removal (Fig. 2e). Administration of DEX after tumour resection increased metastases and precipitated death of MDA-MB 231, PDX and 4T1-bearing animals (Fig. 2f-h). In addition, we inoculated shGR cells into mice and found that DEX treatment upon tumour removal had no impact on overall survival (Extended Data Fig. 3i). These results suggest that shortened survival seen upon GR activation is a result of a direct glucocorticoid effect on cancer cells. This and the observations that GR activation did not increase tumour volume (Extended Data Fig. 3h), enhanced lung metastases growth in both the experimental metastases and orthotopic assays, suggest that GR activation enhances the colonization step of metastases via a cancer cell autonomous mechanism.

We then assessed the consequences of GR activation on signalling pathways. Global proteomic and phosphoproteomic analysis ${ }^{24}$ of lysates from GR activated cells revealed 437 up- and 472 down-regulated proteins and increased phosphorylation in 1556 peptides corresponding to 750 proteins in DEX treated cells compared to controls (Figure 3a, b, Supplementary Table 3). Markers of GR activation and processes such 
as EMT, glucose and nicotinamide metabolism, cytoskeleton organization, and pathways involved in 97 metastases ${ }^{25,26,27,28,29}$ (e.g., EGFR, Hippo) increased upon GR activation (Extended Data Fig. 4a, b)

98 (Extended Data Fig. 4a- d, Supplementary Table 3 and 4). Of the upregulated proteins, there were 63 99 kinases, 6 of which were increased at the RNA level in metastases compared to tumours in MDA-MB 231 100 and PDX models (Figure 3c and Extended Data Fig. 5a-d). Notably, expression of kinases upregulated upon 101 GR activation and in lung metastases was predictive of decreased relapse-free survival in breast cancer 102 patients (Extended Data Fig. 6). These kinases include ROR1 previously implicated in breast cancer $28,30,31$ 103 and we noted that ROR1 signature is associated with decreased survival (Figure 4a). Quantification of 104 ROR1 expression in tumours and matched metastases of MDA-MB 231 and PDX1 model using FACS, 105 confirmed overexpression seen at the transcriptome levels in the lung metastases of MDA-MB 231 model 106 and proteome levels in GR activated cells (Figure 4b, Extended Data Fig. 7a, Supplementary Table 3). We 107 then addressed the effect of ROR1 knockdown (Extended Data Fig. 7b) on metastasis using both 108 experimental metastases and orthotopic assays. Down-regulation of ROR1 using two independent shRNAs, 109 decreased metastasis and prolonged survival in both assays (Figure 4 c, d; Extended Data Fig. 7c). Notably, 110 ROR1 ablation halted GR activation-evoked metastases and prevented the precipitated death of the animals 111 (Figure 4e-g, Extended Data Fig. 7d-f). The data suggest that increased colonization upon GR activation is 112 mediated in part by ROR1. Tumour heterogeneity is one of the major obstacles in treatment of metastatic breast cancers. We 114 show that metastases display distinct phenotypes based on the growth site. We find stress hormone pathway 115 as an important inducer of colonization and death of the animals. We show that ROR1 ablation prevents 116 the deleterious effect of GR activation. Corticosteroids such as DEX are widely used in treatment of breast 117 cancer to decrease the side effects of chemotherapy and treat symptoms related to advanced cancer. Given 118 that cancer cell dissemination has already occurred at the time of primary tumour surgical resection in a 119 substantial number of breast cancer patients ${ }^{16,32}$, and that GR activation fosters colonization at the distant 120 sites, our results call for caution when using corticosteroids in patients. Of note, GR has been shown to 121 evoke adaptive resistance to anti-androgen receptor therapy in prostate tumours ${ }^{33}$. Thus, assessing the 122 effects of stress hormone pathways on metastasis and response to therapy in other cancer types is warranted. 
125 In vivo experiments. All in vivo experiments were performed in accordance with the Swiss animal welfare 126 ordinance and approved by the cantonal veterinary office Basel Stadt. Female severe combined NOD-scid 127 IL2rynull (NSG) and Balb/c animals were maintained in the Friedrich Miescher Institute for Biomedical 128 Research and Department of Biomedicine animal facility in accordance with Swiss guidelines on animal experimentation. MDA-MB 231 cells (10,000 cells) were re-suspended in $40 \mu 1$ Matrigel:PBS (50\%:50\%) and injected in the pre-cleared mammary fat-pad of 4-8 week-old female NSG mice. PDX models were transplanted in the pre-cleared $4^{\text {th }}$ mammary fat-pad of NSG mice, while $4 \mathrm{~T} 1$ cells were injected in the mammary fat-pad of female Balb/c 4-8 week-old mice. Tumours were resected when the longest diameter reached $10 \mathrm{~mm}$ and mice were regularly monitored for the signs of metastatic outgrowth and distress. All the orthotropic experimental procedures (tumour resections and tumour cell implantation) were undertaken in the anesthetized animals according to the animal protocols approved by the cantonal veterinary office Basel Stadt. Experimental metastases assay was performed by injecting 100,000 cells in the tail vein. Upon i.v. injection, in case of MDA-MB 231 cells we performed in vivo bioluminescence imaging to confirm injection and monitor metastatic outgrowth. Bioluminescence imagining was done using IVIS Lumina $X R$ (Caliper LifeSciences) upon injection of luciferin (Biosynth, cat. number L8220).

Cell lines and PDX models. Cell lines MDA-MB 231, 4T1 and HEK293T cells were purchased from the ATCC and cultured according to the ATCC protocol. Cell line identity was confirmed and routinely tested using short tandem repeat (STR) sequencing and all cell lines were routinely tested for mycoplasma contamination. MDA-MB 231 and 4T1 cells were propagated in monolayer cultures in DMEM supplemented with $10 \%$ FCS.

GR activation experiments were performed in monolayer cultures in DMEM supplemented with 2.5\% charcoal stripped FCS (Thermo Fisher Scientific- cat. number 12676029) in presence of water soluble dexamethasone (700 nM, Sigma, D2915) or vehicle.

PDX used for this study were described earlier ${ }^{34,35}$. PDX1, PDX2 and PDX4-11 originate from the primary breast tumours ${ }^{34}$. PDX3 originates from a pleural effusion of a breast cancer patient ${ }^{35}$. Metastatic 150 potential of examined PDX models was analysed by H\&E staining and expression of human specific CD298 151 marker $^{14}$.

152 Lentiviral vectors, lentivirus and infection. For glucocorticoid receptor down-regulation we used 6 153 shRNA constructs (Dharmacon; pGIPZ; V3LHS_404051， V3LHS_404052， V2LHS_239186, 154 V2LHS_82796, V2LHS_82797, V3LHS_326099). ROR1 down-regulation was performed using 4 shRNA 155 constructs (Dharmacon; pTRIPZ; V3THS_349217, V3THS_306714, V3THS_306715, V3THS_240995). 
Non-targeting shRNA (pGIPZ or pTRIPZ) were used as controls. Lentiviral batches were produced using PEI transfection on 293T cells as previously described ${ }^{36}$. The titer of each lentiviral batch was determined in MDA-MB 231 cells. Cells were infected for $8 \mathrm{~h}$ in presence of polybrene $(8 \mu \mathrm{g} / \mathrm{ml})$. Selection with 2 $\mu \mathrm{g} / \mathrm{ml}$ puromycin (Sigma) was applied $48 \mathrm{~h}$ after infection.

Western blot analysis. The cells for immunoblotting were lysed in RIPA buffer (50mM Tris-HCl pH 8 , $150 \mathrm{mM} \mathrm{NaCl}, 1 \% \mathrm{NP}-40,0.5 \%$ sodium deoxycholate, $0.1 \%$ SDS), supplemented with $1 \times$ protease inhibitor cocktail (Complete Mini, Roche), $0.2 \mathrm{mM}$ sodium orthovanadate, $20 \mathrm{mM}$ sodium fluoride, and $1 \mathrm{mM}$ phenylmethylsulfonyl fluoride. Extracted proteins were subjected for BCA protein assay kit (Thermo scientific; cat no: 23227) to measure and equalize their concentration. Whole-cell lysates, immunoprecipitates or nuclear cell lysates $(40 \mu \mathrm{g})$ were subjected to $6 \%$ SDS-PAGE, transferred to PVDF membranes (Immobilon-P, Millipore), and blocked for $1 \mathrm{~h}$ at room temperature with 5\% milk in PBS-0.1\% Tween 20. Membranes were then incubated overnight with antibodies as indicated and exposed to secondary HRP-coupled anti-mouse or -rabbit antibody at 1:5,000-10,000 for $1 \mathrm{~h}$ at room temperature. For each of the blots presented, the results shown are representative of at least three independent experiments. The following antibodies were used: Glucocorticoid receptor antibody (GeneTex; cat. number: GTX101120), pSer GR antibody (TheremoFisher Scientific; cat. number: PA5-17668).

Fluorescence-activated cell sorting. Tumours and matched metastases were mechanically and enzymatically digested using collagenase/hyaluronidase solution (Stemcell technologies; cat. number 07912) at $37{ }^{\circ} \mathrm{C}$. Tumour cells were isolated using FACS based on expression of GFP in MDA-MB 231 and expression of human specific marker CD298 (Biolegend; cat. number 341706). Prior to CTC sorting erythrocytes were eliminated using "Red blood cell lysis buffer" (Sigma- cat. number R7757). Cells were filtered twice through $40-\mu \mathrm{m}$ cell strainers (Falcon) to obtain single cells. FACS was carried out with a BD FACSAria III (Becton Dickinson) using a 70- $\mu$ m nozzle. Single cells were gated on the basis of their forward and side-scatter profiles and pulse-width was used to exclude doublets. Dead cells (DAPI bright) were gated out. Antibodies: APC anti-human CD298 antibody (Biolegend; cat. number: 341706), APC antihuman ROR1 antibody (Biolegend; cat. number: 357805).

RNA preparation, qPCR and sequencing. Isolated cells were sorted in the extraction buffer of Arctutus ${ }^{\circledR}$ PicoPure ${ }^{\circledR}$ RNA Isolation Kit (Cat. number 12204-01) and mRNA was isolated using manufacturer protocol. RNA was depleted of rRNA using the Ribo-Zero Magnetic Kit (MRZ11124C) from Epicenter and column purified with the RNA Cleanup \& Concentrator from Zymo Research. RNA integrity was measured on an Agilent 2100 Bioanalyzer using RNA Pico reagents (Agilent Technologies). The library was prepared using the ScriptSeq v2 RNA-Seq Library Preparation Kit (Epicentre). Library quality was measured on an Agilent 2100 Bioanalyzer for product size and concentration. Single-end libraries were 
sequenced by an Illumina HiSeq 2500 (50-nucleotide read length). Quantitative PCR analysis was done upon mRNA isolation (Qiagene, RNeasy Plus Mini kit, cat. number 74136). We used $1 \mu \mathrm{g}$ of mRNA for cDNA generation (BioRad, iScript cDNA synthesis kit, cat. number 170-8891) and IDT master mix. HPRT1 was used as a housekeeping gene (IDT Hs.PT.58v.45621572). IDT predesigned qPCR assay Ids: NR3C1: Hs.PT.58.27480377, ROR1: Hs.PT.58.39481678, FN1: Hs.PT.58.40005963, KLF9: Hs.PT.56A.15636661， SNAI2: Hs.PT.58.177250059， VIM: Hs.PT.58.38906895, POU5F1: Hs.PT.58.14494169g, MT2A: Hs.PT.5046709.g, and taqman probes (Thermo Fisher Scientific) ANKRD1: Hs00923599_m1, ID3: Hs00954037_g1. All measurements were performed in duplicates and triplicates as previously described ${ }^{36}$. The arithmetic mean of the $\mathrm{Ct}$ values was used for calculations: target gene mean $\mathrm{Ct}$ values were normalized to the respective housekeeping genes (HPRT1), mean $\mathrm{Ct}$ values (internal reference gene, $\mathrm{Ct}$ ), and then to the experimental control. The values obtained were $2-\Delta \Delta \mathrm{Ct}$ expressed as fold changes in regulation compared to the experimental control using the $2-\Delta \Delta \mathrm{Ct}$ method of relative quantification.

Quantification of cortisol and corticosterone in mouse plasma. Cortisol ( $\geq 99 \%)$, corticosterone ( $\geq 98.5 \%$ ), and formic acid ( $\geq 98 \%$ ) were purchased from Sigma-Aldrich (Buchs, Switzerland). Diazepam ( $\geq 98 \%$ ) was acquired from Toronto Research Chemicals (Toronto, Canada). Oasis HLB cartridges (1 cc, $30 \mathrm{mg}$ ) were from Waters (Milford, MA). Solvents of LC-MS or higher purity grade were used. Stock solutions of compounds were prepared in DMSO or methanol and stored at $-20{ }^{\circ} \mathrm{C}$ until use.

Mice blood samples were collected in EDTA coated tubes and mice plasma was prepared by centrifugation for 15 minutes at 2,000 g. Plasma samples were stored at $-80{ }^{\circ} \mathrm{C}$ until analysis. To precipitate proteins and extract cortisol, cortisone, and corticosterone from mice plasma, $50 \mu \mathrm{L}$ of plasma was mixed with $950 \mu \mathrm{L}$ of acetonitrile. Samples were shaken for $30 \mathrm{~min}$ at $10{ }^{\circ} \mathrm{C}$, placed at $-20{ }^{\circ} \mathrm{C}$ for $30 \mathrm{~min}$, and then centrifuged for $20 \mathrm{~min}$ at $24,000 \mathrm{~g}$ at $5{ }^{\circ} \mathrm{C}$. Supernatants were separated, while remaining pellets were reconstituted in $100 \mu \mathrm{L}$ of water and extracted for the second time with $900 \mu \mathrm{L}$ of acetonitrile. After second centrifugation (20 min at $24,000 \mathrm{~g}$ and $5{ }^{\circ} \mathrm{C}$ ), supernatants from two plasma extraction steps were combined and concentrated in vacuum to approximate volume of $100 \mu \mathrm{L}$. Oasis HLB solid-phase extraction cartridges (SPE; $1 \mathrm{cc}, 30 \mathrm{mg}$ ) were activated with acetonitrile $(1 \mathrm{~mL}$ ) and conditioned with $5 \%$ acetonitrile in $0.1 \%$ formic acid in water $(1 \mathrm{~mL})$. Plasma extracts were applied to the SPE cartridges and washed with $0.1 \%$ formic acid in water $(3 \times 1 \mathrm{~mL})$. Analytes were eluted from SPE cartridges with acetonitrile $(3 \times 0.5 \mathrm{~mL})$, samples were concentrated in vacuum, spiked with solution of internal standard (500 nM diazepam), and reconstructed up to $500 \mu \mathrm{L}$ with HPLC mobile phase.

For quantification of cortisol, cortisone, and corticosterone in mice plasma, samples were analyzed with Quattro Ultima triple-quadruple mass spectrometer equipped with an electro-spray source (Waters, Milford, 
MA) and coupled to an Agilent 1200 HPLC system (Agilent, Santa Clara, CA). Analytical column was a HALO C18 $(100 \times 2.1 \mathrm{~mm}, 2.7 \mu \mathrm{m}$; Advanced Materials Technology, Wilmington, Delaware). The column flow rate and temperature were $400 \mu \mathrm{L} \cdot \mathrm{min}^{-1}$ and $50{ }^{\circ} \mathrm{C}$, respectively. Eluents A and B were $0.1 \%$ formic acid in water and acetonitrile, respectively. Gradient elution was as follows: $0-1 \mathrm{~min}, 5 \% \mathrm{~B} ; 1-10 \mathrm{~min}$, $5 \rightarrow 100 \% \mathrm{~B} ; 10-11 \mathrm{~min}, 100 \% \mathrm{~B}, 11-12 \mathrm{~min}, 100 \rightarrow 5 \% \mathrm{~B} ; 12-15 \mathrm{~min}, 5 \% \mathrm{~B}$. Source and desolvation temperatures were 140 and $240{ }^{\circ} \mathrm{C}$, respectively, whereas cone and desolvation gas flows were 50 and 500 $\mathrm{L}$ hour ${ }^{-1}$. Capillary voltage was $2.5 \mathrm{kV}$, cone voltage was $70 \mathrm{~V}$, and collision energy was 16-28 eV. Analyte quantification was performed in the positive ionization mode, relative to internal standard (diazepam), using a multiple-reaction monitoring mode. Following transitions were used $(\mathrm{m} / \mathrm{z})$ : $\operatorname{cortisol}(363.1 \rightarrow 121.1,327.2$, $309.2,345.2)$, cortisone $(361.1 \rightarrow 163.1)$, corticosterone $(347.1 \rightarrow 329.2,311.2,293.2,121.1)$, and diazepam $(285 \rightarrow 222,228,257$; internal standard). Calibration curves were prepared by spiking of mobile phase with authentic metabolite standards in the concentration range of $0.8-1000 \mathrm{nM}$. Chromatograms were analyzed with MassLynx 4.1 software (Waters, Milford, MA). Lower limits of detection (LLOD) and quantification (LLOQ) in mouse plasma samples were assessed based on signal-to-noise ratios of 3 and 10, respectively. Observed LLOD (lower limit of detection) for cortisol, cortisone, and corticosterone were 1.46, 0.25, and $0.85 \mathrm{ng} / \mathrm{mL}$. Observed LLOQ (lower limit of quantification) for cortisol, cortisone, and corticosterone were $4.56,0.84$, and $2.55 \mathrm{ng} / \mathrm{mL}$.

Proteomics and phosphoproteomics analysis. MDA-MB 231 cells propagated for seven days in charcoal stripped FCS in presence of dexamethasone or vehicle were mechanically detached, washed and snap frozen. The results normalized for multiple testing by Benjamin Hochberg correction.

Immunohistochemistry. Tissue was fixed in FormalFix for $24 \mathrm{~h}$ at $4{ }^{\circ} \mathrm{C}$, washed with $70 \%$ ethanol, embedded in paraffin, and 3- $\mu \mathrm{m}$ sections prepared and processed for haematoxylin and eosin staining and immunohistochemistry.

Computational analysis. Sequenced reads were aligned against Human Feb.2009 (GRCh37/hg19)(hg19) and analysis was performed using FMI Galaxy platform ${ }^{37}$ (QuasR ${ }^{38}$, R/Bioconductor). Differential gene expression was determined using edgeR ${ }^{39}$, a cut-off of a linear fold change $\geq 2$ and adjusted $F D R \leq 0.05$ (corrected with the Benjamini-Hochberg algorithm method) was used. Integrated system for motif activity response analysis (ISMARA) was performed as described ${ }^{36}$. For the transcription-factor binding-site enrichment, we used oPOSSUM (v1) (http://opossum.cisreg.ca/oPOSSUM3/). We used Ingenuity Pathway Analysis (IPA) for the search for the Upstream Regulators. MetaCore pathway analysis was used for the analysis of phosphoproteomic data, while we used String ${ }^{40}$ (https://stringdb.org/cgi/input.pl?UserId=input_page_show_search=on) for the analysis of proteomic data. GSEA was performed using the JAVA application from the Broad Institute v2.0 (http://www.broadinstitute. org/gsea). 
We used cBioPortal $^{41,42}$ for the GR expression correlation study with publicly available data ${ }^{21,22}$. RFS, DMFS and PPS were generated using 2017 version of KMplotter $^{43}$ (http://kmplot.com/analysis/index.php?p=service\&cancer=breast), while ROR1 and kinase based signature relapse-free survival was generated using g-2-o platform ${ }^{44}$ (http://www.g-2-o.com/?q=G2OBreast).

Statistical data analysis. Standard laboratory practice randomization procedure was used for cell line groups and animals of the same age and sex. The investigators were not blinded to allocation during experiments and outcome assessment. The number of mice was calculated by performing power analysis using data from small pilot experiments. Values represent the means \pm s.d. $P$ values were determined using unpaired two-tailed $t$-tests and statistical significance was set at $P=0.05$. The variance was similar between groups that we compared. Experimental replicates are independent experiments. Technical replicates are tests or assays run on the same sample multiple times. Data were tested for normal distribution and Student's $t$-tests (if normally distributed) or nonparametric Mann-Whitney U/Wilcoxon-tests were applied unless stated otherwise. Kaplan-Meier plots were generated using the survival calculation tool from Graphpad Prism and significance was calculated using the log-rank test at $P<0.05$.

Data availability. Transcriptomics, proteomics and phosphoproteomics data are available upon request during the review process.

\section{References}

1. Almendro, V., Marusyk, A. \& Polyak, K. Cellular Heterogeneity and Molecular Evolution in Cancer. Annu. Rev. Pathol. Mech. Dis. 8, 277-302 (2013).

2. Koren, S. \& Bentires-Alj, M. Breast Tumor Heterogeneity: Source of Fitness, Hurdle for Therapy. Mol. Cell 60, 537-546 (2015).

3. Polzer, B. \& Klein, C. A. Metastasis Awakening: The challenges of targeting minimal residual cancer. Nat. Med. 19, 274-275 (2013).

4. Hanahan, D. \& Weinberg, R. A. Hallmarks of Cancer: The Next Generation. Cell 144, 646-674 (2011).

5. Weinberg, R. A. Robert A. The biology of cancer. (Gerald Science, 2014).

6. Marusyk, A., Almendro, V. \& Polyak, K. Intra-tumour heterogeneity: a looking glass for cancer? Nat. Rev. Cancer 12, 323-334 (2012).

7. Chaffer, C. L., San Juan, B. P., Lim, E. \& Weinberg, R. A. EMT, cell plasticity and metastasis. Cancer Metastasis Rev. 35, 645-654 (2016). 
8. Meacham, C. E. \& Morrison, S. J. Tumour heterogeneity and cancer cell plasticity. Nature (2013). doi:10.1038/nature12624

9. Vanharanta, S. \& Massagué, J. Origins of Metastatic Traits. Cancer Cell 24, 410-421 (2013).

10. Obenauf, A. C. \& Massagué, J. Surviving at a distance: organ specific metastasis. Trends in cancer 1, 76-91 (2015).

11. Stefanovic, S. et al. Tumor biomarker conversion between primary and metastatic breast cancer: mRNA assessment and its concordance with immunohistochemistry. Oncotarget 8, 51416-51428 (2017).

12. Lindström, L. S. et al. Clinically used breast cancer markers such as estrogen receptor, progesterone receptor, and human epidermal growth factor receptor 2 are unstable throughout tumor progression. J. Clin. Oncol. 30, 2601-8 (2012).

13. Alizadeh, A. A. et al. Toward understanding and exploiting tumor heterogeneity. Nat. Med. 21, 846-853 (2015).

14. Lawson, D. A. et al. Single-cell analysis reveals a stem-cell program in human metastatic breast cancer cells. Nature 526, 131-5 (2015).

15. Balwierz, P. J. et al. ISMARA: automated modeling of genomic signals as a democracy of regulatory motifs. Genome Res. 24, 869-884 (2014).

16. Hosseini, H. et al. Early dissemination seeds metastasis in breast cancer. Nature 540, 552-558 (2016).

17. Van Der Pompe, G., Antoni, M. H. \& Heijnen, C. J. Elevated basal cortisol levels and attenuated ACTH and cortisol responses to a behavioral challenge in women with metastatic breast cancer. Psychoneuroendocrinology 21, 361-374 (1996).

18. Sephton, S. E. Diurnal Cortisol Rhythm as a Predictor of Breast Cancer Survival. J. Natl. Cancer Inst. 92, 994-1000 (2000).

19. Chen, Z. et al. Ligand-dependent genomic function of glucocorticoid receptor in triple-negative breast cancer. Nat. Commun. 6, 8323 (2015).

20. Shibue, T. \& Weinberg, R. A. EMT, CSCs, and drug resistance: the mechanistic link and clinical implications. Nat. Rev. Clin. Oncol. 14, 611-629 (2017).

21. Curtis, C. et al. The genomic and transcriptomic architecture of 2,000 breast tumours reveals novel 
subgroups. Nature 486, 346-52 (2012).

315 22. Pereira, B. et al. The somatic mutation profiles of 2,433 breast cancers refines their genomic and transcriptomic landscapes. Nat. Commun. 7, 11479 (2016).

317 23. Saxena, M. \& Christofori, G. Rebuilding cancer metastasis in the mouse. Mol. Oncol. 7, 283-296 (2013).

319 24. Ahrné, E. et al. Evaluation and Improvement of Quantification Accuracy in Isobaric Mass TagBased Protein Quantification Experiments. J. Proteome Res. 15, 2537-47 (2016).

321

322

323

324

325

326

25. Banerjee, K. \& Resat, H. Constitutive activation of STAT3 in breast cancer cells: A review. Int. J. Cancer 138, 2570-2578 (2016).

26. Marotta, L. L. C. et al. The JAK2/STAT3 signaling pathway is required for growth of $\mathrm{CD} 44^{+} \mathrm{CD} 24^{-}$stem cell-like breast cancer cells in human tumors. J. Clin. Invest. 121, 2723-35 (2011).

27. Liu, J. et al. Synaptopodin-2 suppresses metastasis of triple-negative breast cancer via inhibition of YAP/TAZ activity. J. Pathol. 244, 71-83 (2018).

28. Li, C. et al. A ROR1-HER3-IncRNA signalling axis modulates the Hippo-YAP pathway to regulate bone metastasis. Nat. Cell Biol. 19, 106-119 (2017).

29. Dearth, R. K. et al. Mammary tumorigenesis and metastasis caused by overexpression of insulin receptor substrate 1 (IRS-1) or IRS-2. Mol. Cell. Biol. 26, 9302-14 (2006).

30. Cui, B. et al. Targeting ROR1 inhibits epithelial-mesenchymal transition and metastasis. Cancer Res. 73, 3649-60 (2013).

31. Chien, H.-P. et al. Expression of ROR1 has prognostic significance in triple negative breast cancer. Virchows Arch. 468, 589-595 (2016).

32. Braun, S. et al. A Pooled Analysis of Bone Marrow Micrometastasis in Breast Cancer. N. Engl. J. Med. 353, 793-802 (2005).

33. Arora, V. K. et al. Glucocorticoid Receptor Confers Resistance to Antiandrogens by Bypassing Androgen Receptor Blockade. Cell 155, 1309-1322 (2013).

34. Gao, H. et al. High-throughput screening using patient-derived tumor xenografts to predict clinical trial drug response. Nat. Med. 21, 1318-1325 (2015). 
35. DeRose, Y. S. et al. Tumor grafts derived from women with breast cancer authentically reflect tumor pathology, growth, metastasis and disease outcomes. Nat. Med. 17, 1514-1520 (2011).

36. Britschgi, A. et al. The Hippo kinases LATS1 and 2 control human breast cell fate via crosstalk with ERa. Nat. Publ. Gr. 541, 4-1 (2017).

37. Afgan, E. et al. The Galaxy platform for accessible, reproducible and collaborative biomedical analyses: 2016 update. Nucleic Acids Res. 44, W3-W10 (2016).

38. Gaidatzis, D., Lerch, A., Hahne, F. \& Stadler, M. B. QuasR: quantification and annotation of short reads in R. Bioinformatics 31, 1130-1132 (2015).

39. Robinson, M. D., McCarthy, D. J. \& Smyth, G. K. edgeR: a Bioconductor package for differential expression analysis of digital gene expression data. Bioinformatics 26, 139-140 (2010).

40. Szklarczyk, D. et al. The STRING database in 2017: quality-controlled protein-protein association networks, made broadly accessible. Nucleic Acids Res. 45, D362-D368 (2017).

41. Gao, J. et al. Integrative Analysis of Complex Cancer Genomics and Clinical Profiles Using the cBioPortal. Sci. Signal. 6, pl1-pl1 (2013).

42. Cerami, E. et al. The cBio cancer genomics portal: an open platform for exploring multidimensional cancer genomics data. Cancer Discov. 2, 401-4 (2012).

43. Györffy, B. et al. An online survival analysis tool to rapidly assess the effect of 22,277 genes on breast cancer prognosis using microarray data of 1,809 patients. Breast Cancer Res. Treat. 123, 725-731 (2010).

44. Pongor, L. et al. A genome-wide approach to link genotype to clinical outcome by utilizing next generation sequencing and gene chip data of 6,697 breast cancer patients. (2011). doi:10.1186/s13073-015-0228-1

\section{Acknowledgments}

We thank members of the Bentires-Alj laboratory for advice and discussions. The authors are grateful to Alana L. Welm, University of Utah for PDX3 and PDX12-16 models. Tissue samples corresponding to PDX1, 2, 4-11 were provided by the Cooperative Human Tissue Network which is funded by the National Cancer Institute. Other investigators may have received specimens from the same subjects. We are grateful for the support of FMI, DBM and Biozentrum core facilities. Research in the Bentires-Alj laboratory is 
371 supported by the Swiss Initiative for Systems Biology- SystemsX, the European Research Council, the

372 Swiss National Science Foundation, the Krebsliga Beider Basel, the Swiss Cancer League and the

373 Department of Surgery of the University Hospital Basel.

\section{Author contributions}

375 M.O. conceived the study, designed and performed all the experiments, analysed the data, interpreted the 376 results and wrote the manuscript. B.H. performed experiments on gene expression and helped with shROR1 377 experiments and animal experiments, analysed the data and interpreted the results. N.M. established method 378 and measured stress hormones levels in plasma, analysed the data and interpreted the results. J.P.C. 379 designed experiments, analysed the data and interpreted the results. S.M. and S.B. performed 380 histopathological analysis of utilized PDX models, analysed the data and interpreted the results. R.O. 381 characterized metastatic potential of PDX models analysed the data and interpreted the results. H.K. 382 performed FACS experiments, analysed the data and interpreted the results. H-R.H. performed 383 transcriptomics data analysis and interpreted the results. A.S. performed proteomics and 384 phosphoproteomics experiments, analysed the data and interpreted the results. M.B-A. conceived the study, 385 designed the experiments and interpreted the results. All authors read and approved the final manuscript.

\section{Author information}

387 The authors declare no competing financial interests.

388 Materials and correspondence

389 Correspondence and requests for materials should be addressed to Mohamed Bentires-Alj, M.Bentires390 Alj@unibas.ch.

391 This manuscript contains 4 Figures, 7 Extended Data Figure, 4 Supplementary Tables. 


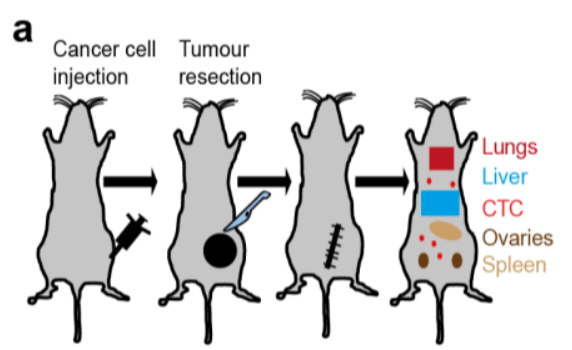

d

\begin{tabular}{|l|c|c|}
\hline Upstream regulator & Activation Z-score & $P$-value of overlap \\
\hline Dexamethasone & 2.820 & $6.76 \mathrm{e}-16$ \\
\hline Triamcinolone acetonide & 2.635 & $1.27 \mathrm{e}-09$ \\
\hline R5020 & 2.630 & $3.89 \mathrm{e}-09$ \\
\hline GR & 2.279 & $1.09 \mathrm{e}-09$ \\
\hline
\end{tabular}

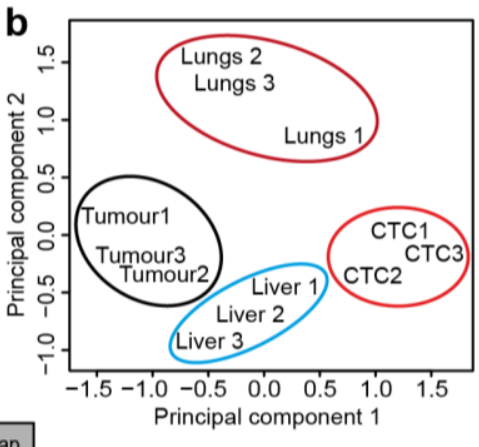

f

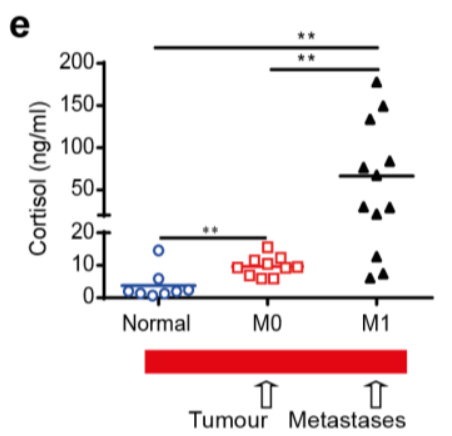

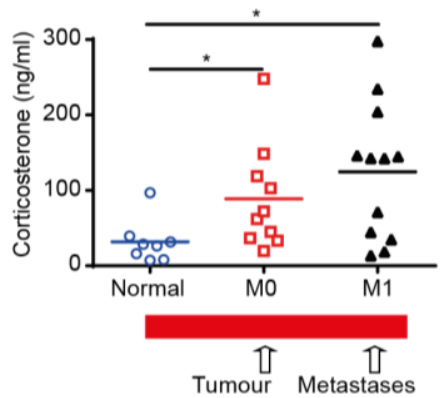

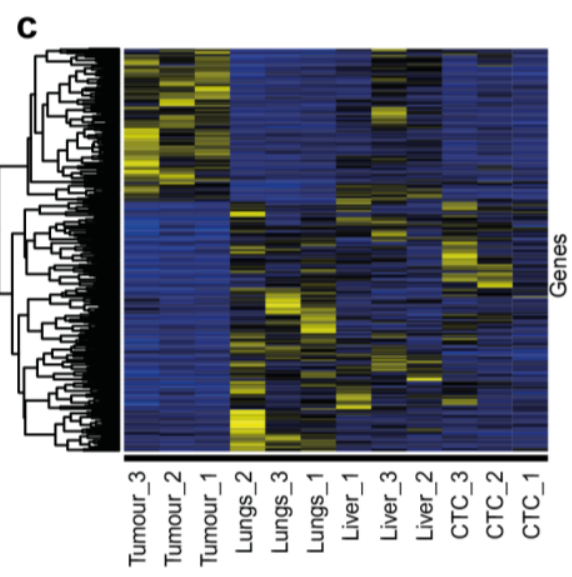

g

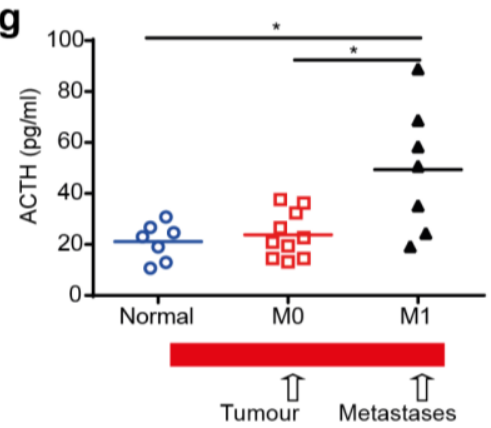

Figure 1. Increase of GR activation in distant metastases. a, Experimental design for the study of tumour heterogeneity in PDX and MDA-MB 231 model. MDA-MB 231 cells were injected in the mammary fatpad of female NSG mice. Cells isolated from the "tumour" (tumour cells found in the mammary fat-pad after orthotopic injection/transplantation) or matched "metastases" (cancer cells found in the distant sites such as lungs, liver etc. weeks after tumour resection) were sorted by FACS. b, Principal component analysis of tumours and matched metastases in MDA-MB 231 model clustered transcriptional profiles based on the site of metastases. The names in PCA plot represent tumour cells isolated from the indicated site, while the numbers within the names indicate a mouse; $n=3$ biological replicates. c, Heatmap of differentially expressed genes between tumours and lung metastases in MDA-MB 231 model; 280 genes yellow $=$ up-regulation, blue $=$ down-regulation, fold change $\geq 2, \operatorname{FDR}<0.05, n=3$ biological replicates. d, Ingenuity Pathway Analysis revealed GR and GR ligands as the Upstream Regulators of the lung metastatic phenotype, $n=3$ biological replicates. Increased levels of $\mathbf{e}$, cortisol, $\mathbf{f}$, corticosterone and $\mathbf{g}$, ACTH in the plasma of animals prior to tumour resection and animals with the distant metastases. Mean and single data points are represented, $n=8$-12 mice, biological replicates, two-tailed Student's $t$-test, $* P<0.05$, $* * P<0.01$. 
a

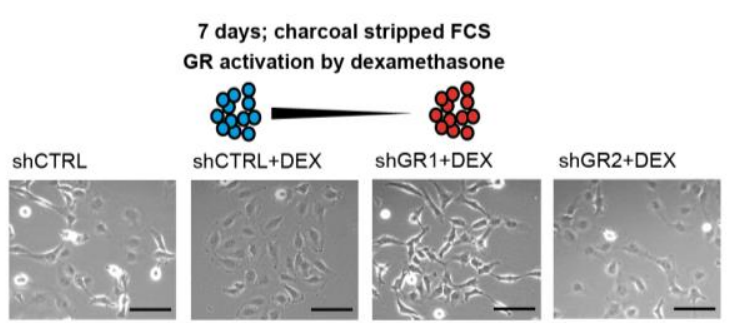

c

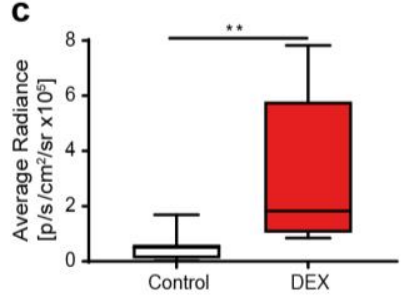

e

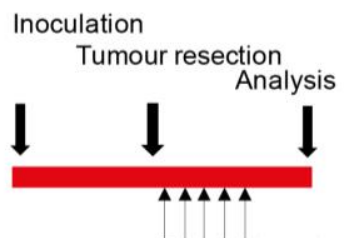

Dex treatment

h

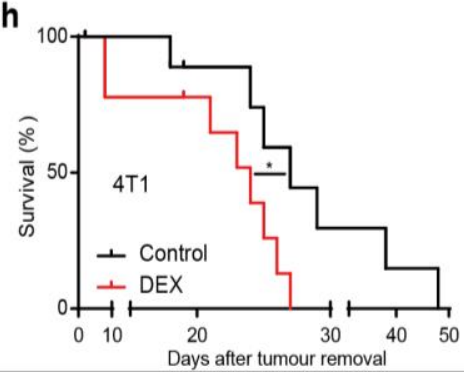

b

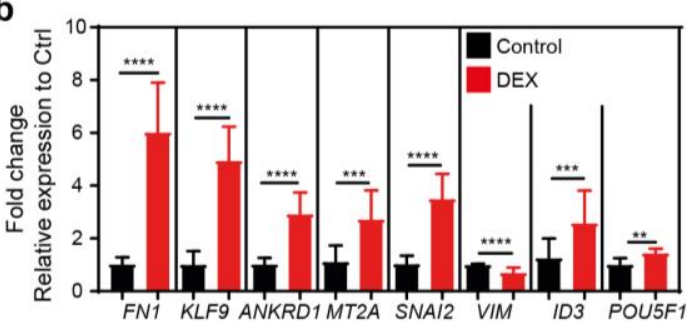

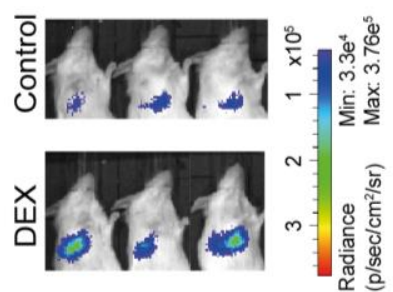

f

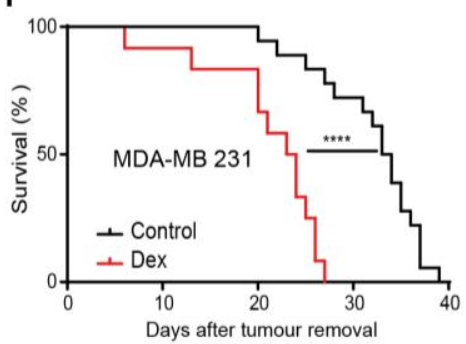

d

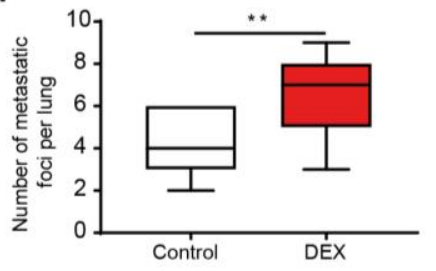

g

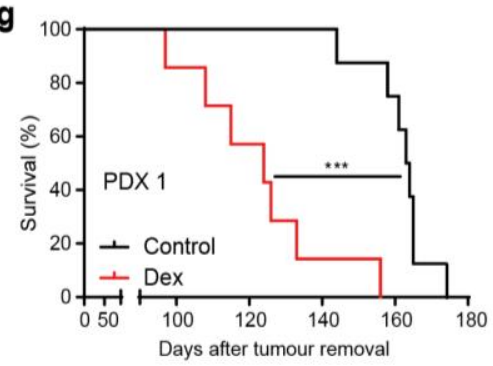

Figure 2. GR activation escalates metastatic colonization and reduces survival. a, MDA-MB 231 cells were propagated in the presence of dexamethasone (DEX) for 7 days, scale bar $200 \mu \mathrm{m}$. b. Expression of

412 GR targets after prolonged GR activation by DEX was assessed by qPCR. Data are mean \pm s.d., $n=6$

413 biological replicates each measured in 2 experimental replicates; two-tailed Student's $t$-test, $* * P<0.01$,

$414 * * * P<0.001, * * * * P<0.0001$. c, Box plot- GR activation increased lung colonization upon i.v. injection in

415 MDA-MB 231 model. Right, bioluminescence imaging two weeks after cell injection, $n=7$ mice and d, 4T1

416 model $n=9$ mice, ${ }^{*} * P<0.01$, two-tailed Student's $t$-test. e-h, Administration of DEX after tumour removal

417 in orthotopic models reduced survival in $\mathbf{f}$, MDA-MB $231 n=12-18$ mice, pooled data from 3 independent

418 experiments, $\mathbf{g}, \mathrm{PDX} 1, n=7-8$ mice; and $\mathbf{h}, 4 \mathrm{~T} 1$ model, $n=9$ mice, $* P<0.05$, $* * * P<0.001$, $* * * * P<0.0001$,

419 Log-rank test. 
a

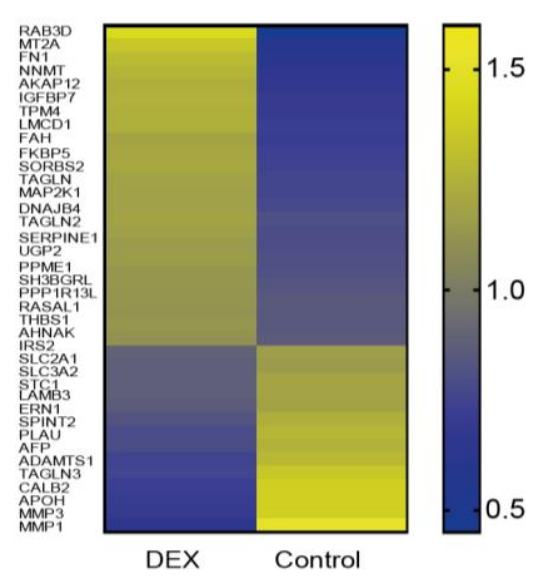

b

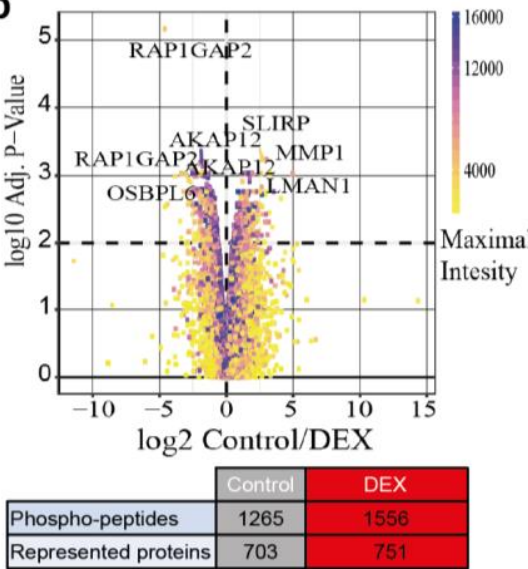

C

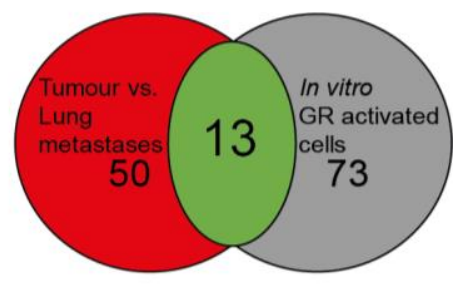

\begin{tabular}{|c|c|c|}
\hline ROR1* & EIF2AK3 & PGK1 \\
\hline MYLK* & TGFBR2* & PRKCA \\
\hline CDK1 & HK2 & ADK \\
\hline MELK & WNK1 & \multicolumn{1}{|}{} \\
\cline { 1 - 2 } TTK & PDK1 & \multicolumn{1}{|c}{} \\
\cline { 1 - 2 } & \multicolumn{2}{|l}{}
\end{tabular}

421 Figure 3. GR activation induces signalling networks and protein kinases implicated in breast cancer

422 progression. a, Heatmap of differentially expressed proteins between MDA-MB 231 cells propagated in 423 presence of DEX ( $n=3$ "control" biological replicates, $n=4$ "DEX" biological replicates, median expression, 424 FDR $<0.01$; yellow = up-regulation, blue= down-regulation). $\mathbf{b}$, Differential peptide phosphorylation of GR 425 activated and control MDA-MB 231 cells $(n=3$ control biological replicates, $n=4$ DEX biological replicates, 426 median expression, FDR $<0.05$ ). c, Overlap of differentially regulated protein kinases in the lung metastases of MDA-MB 231 model and in vitro propagated cells. 
a

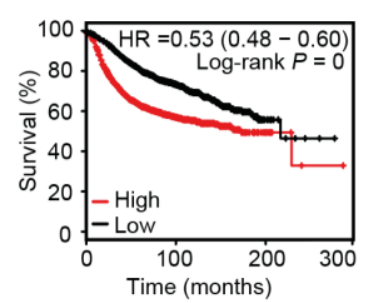

C

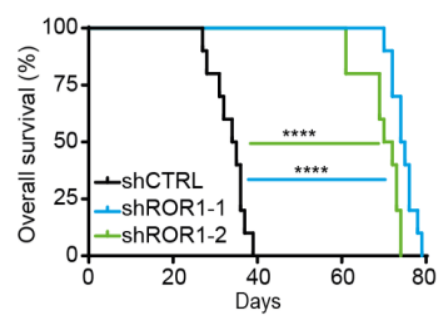

e

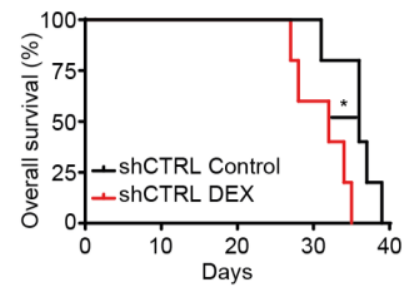

d b
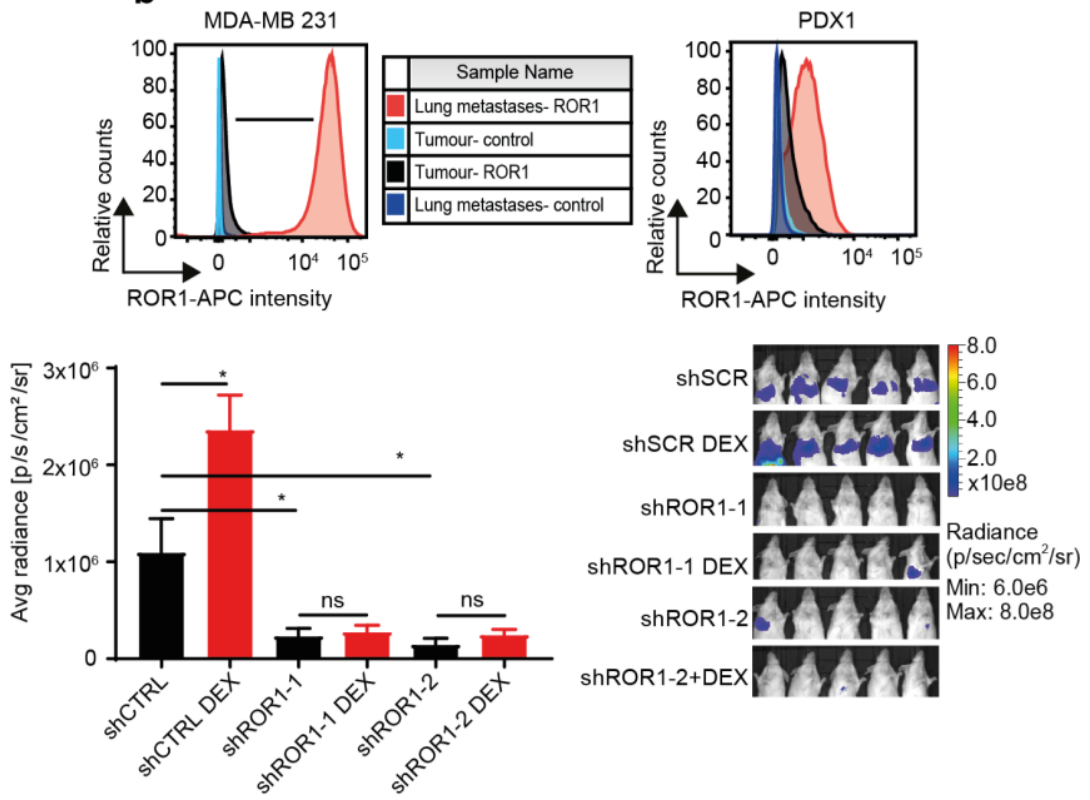

f

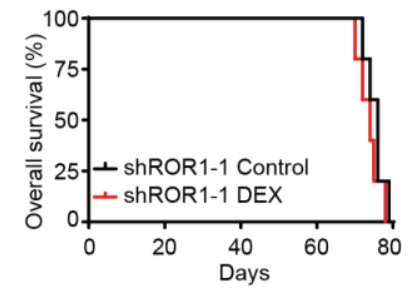

g

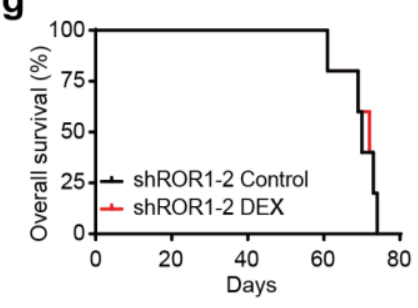

Figure 4. ROR1 mediates GR induced lung metastatic colonization. a, Relapse-free survival of patients with ROR1 signature (G-2-0 Kaplan-Meier), n=4029, Log-rank test. b, Flow cytometry of ROR1 expression in tumour and matched metastases of MDA-MB 231 and PDX1, $n=3$ biological replicates. c, Kaplan-Meier survival analysis of animals inoculated i.v. with control or shROR1 MDA-MB 231 cells, $n=10$ mice per group, $* * * * P<0.0001$, Log-rank test. d, Experimental metastases assay (i.v.) and in vivo

434 bioluminescence imaging of animals inoculated with GR activated or control MDA-MB 231 down435 regulated for ROR1 or shSCR, ${ }^{*} P<0.05$, $n s=$ non-significant, two-tailed Student's $t$-test. e-g, Kaplan-Meier survival analysis of animals inoculated i.v. with e, shSCR, f, shROR1-1 and g, shROR1-2 MDA-MB 231

437 cells propagated in presence of DEX or vehicle, $n=5$ mice per group, $* P<0.05$, Log-rank test. 
a

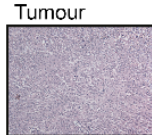

Spleen
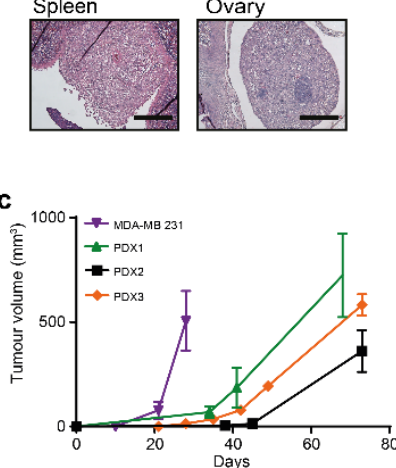

Tumour

Lung metastases

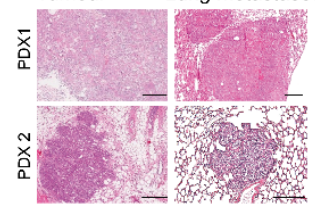

g
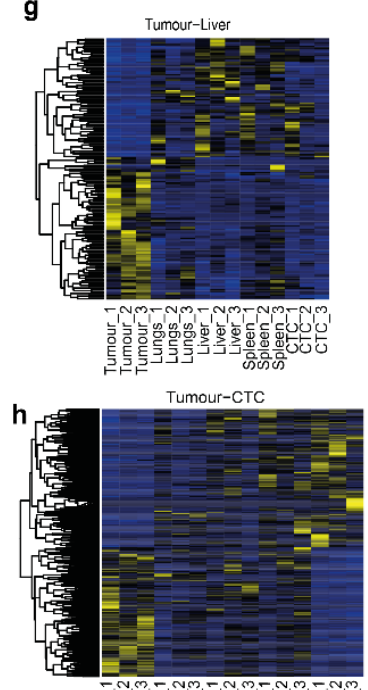

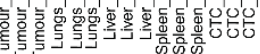

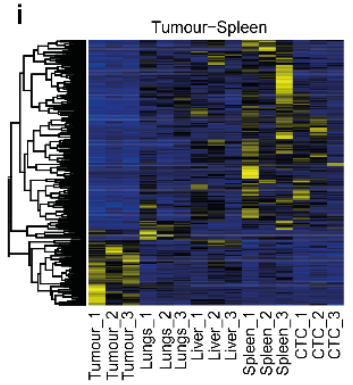

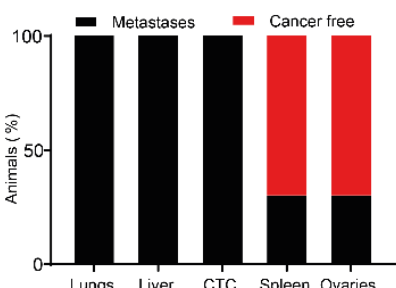

d
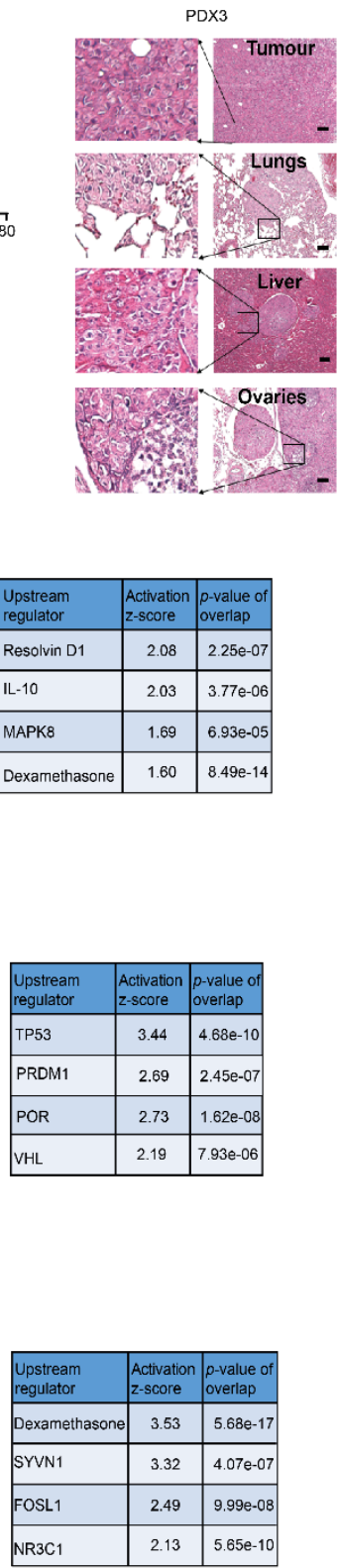

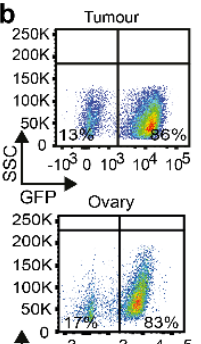

of $-10^{3} 010^{3} 10^{4} 10^{5}$ (i)
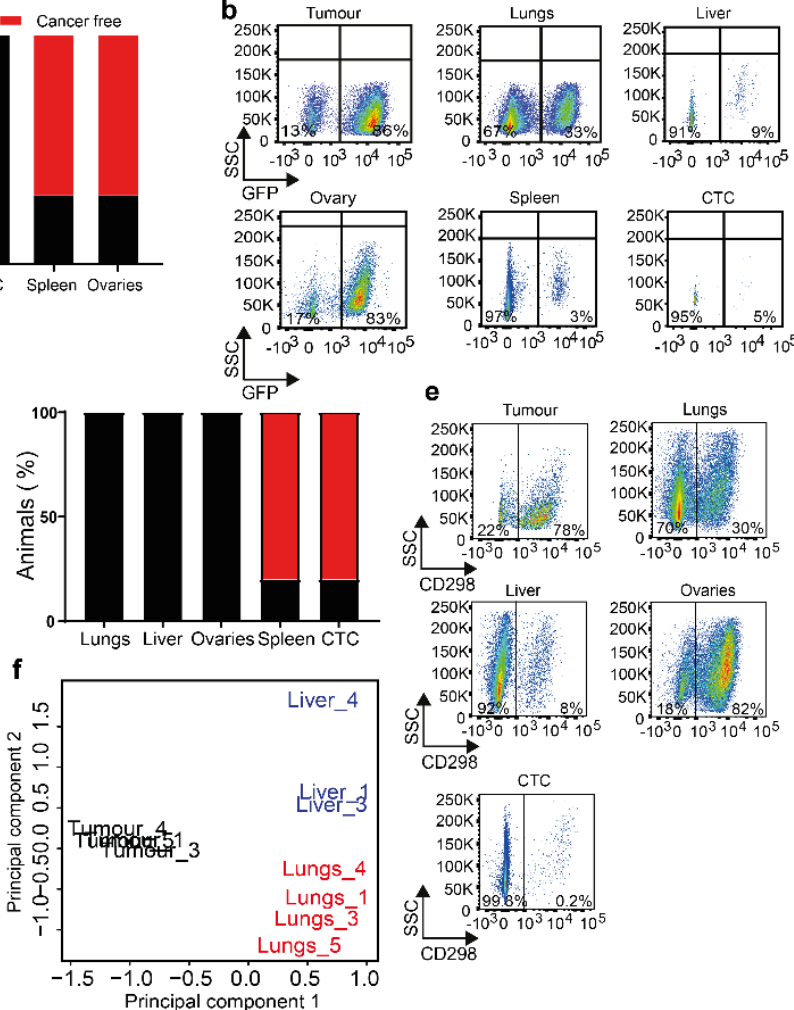
$\longrightarrow$ CD298 CTC

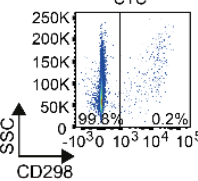
Principal component

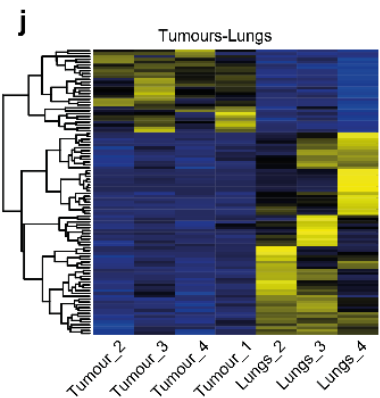

k

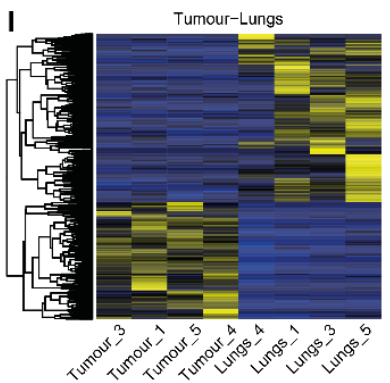

m

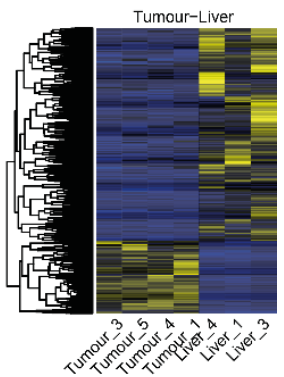


441 and eosin staining), scale bar $200 \mu \mathrm{m}$. Right- frequency of metastases detected in the distant organs upon 442 tumour resection, $n=10$ mice from independent experiments. b, FACS analysis of organs affected with 443 distant metastases of MDA-MB 231 model, $n=10$ mice from independent experiments. c, Tumour growth 444 kinetics after orthotopic transplantation in MDA-MB 231 and PDX models, $n=5$ biological replicates. 445 Lower; Tumour and matched lung metastases in PDX1 and PDX2 models (hematoxylin and eosin staining), 446 scale bar $200 \mu \mathrm{m}$. d, Tumour and matched lung, liver, ovary metastases in PDX3 model (hematoxylin and 447 eosin staining), scale bar $200 \mu \mathrm{m}$. Right- Frequency of metastases detected in the distant organs upon 448 tumour resection, $n=10$ mice from independent experiments. e, FACS analysis of tumour and organs with 449 distant matched metastases in PDX3 model, $n=5$ biological replicates. f, Principal component analysis of 450 tumours and matched metastases in PDX3, n=3-4 biological replicates. g, MDA-MB 231- Heatmap of 451 differentially expressed genes between tumour and liver metastases; right- Upstream regulator analysis 452 (Ingenuity Pathway Analysis- IPA), $n=3$ biological replicates, fold change $\geq 2$, FDR $<0.05$; $\mathbf{h}$, MDA-MB 453 231- Heatmap of differentially expressed genes between tumour and CTC; right- Upstream regulator 454 analysis (Ingenuity Pathway Analysis- IPA), $n=3$ biological replicates, fold change $\geq 2$, FDR $<0.05$; i, MDA455 MB 231- Heatmap of differentially expressed genes between tumour and Spleen; right- Upstream regulator 456 analysis (Ingenuity Pathway Analysis- IPA), $n=3$ biological replicates, fold change $\geq 2$, FDR $<0.05 ; \mathbf{j}$, 457 PDX1- Heatmap of differentially expressed genes between tumour and lung metastases, $n=3-4$ biological 458 replicates, fold change $\geq 2$, FDR $<0.05$. k, PDX2- Heatmap of differentially expressed genes between tumour 459 and lung metastases, $n=4$ biological replicates, fold change $\geq 2$, FDR $<0.05$. 1, PDX3- Heatmap of 460 differentially expressed genes between tumour and lung metastases, $n=4$ biological replicates, fold change $461 \geq 2$, FDR $<0.05$. m, PDX3- Heatmap of differentially expressed genes between tumour and liver metastases, $462 n=4$ biological replicates, fold change $\geq 2, \mathrm{FDR}<0.05$. 

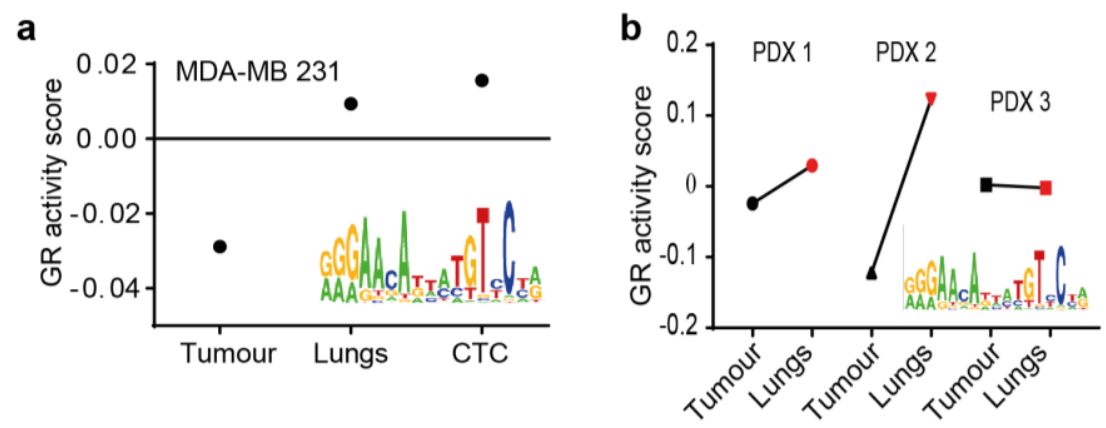

C

\begin{tabular}{|l|c|c|c|c|}
\hline \multicolumn{1}{|c|}{ GR } & Target gene hits & Target TFBS hits & z-score & Fisher score \\
\hline MDA-MB 231 & 25 & 32 & 7.819 & 6.771 \\
\hline PDX 1 & 42 & 55 & 16.522 & 15.046 \\
\hline PDX2 & 44 & 72 & 3.001 & 16.591 \\
\hline PDX 3 & 42 & 53 & 4.802 & 5.706 \\
\hline MMTV-HER2 ${ }^{16}$ & 24 & 30 & 4.382 & 2.564 \\
\hline
\end{tabular}

d
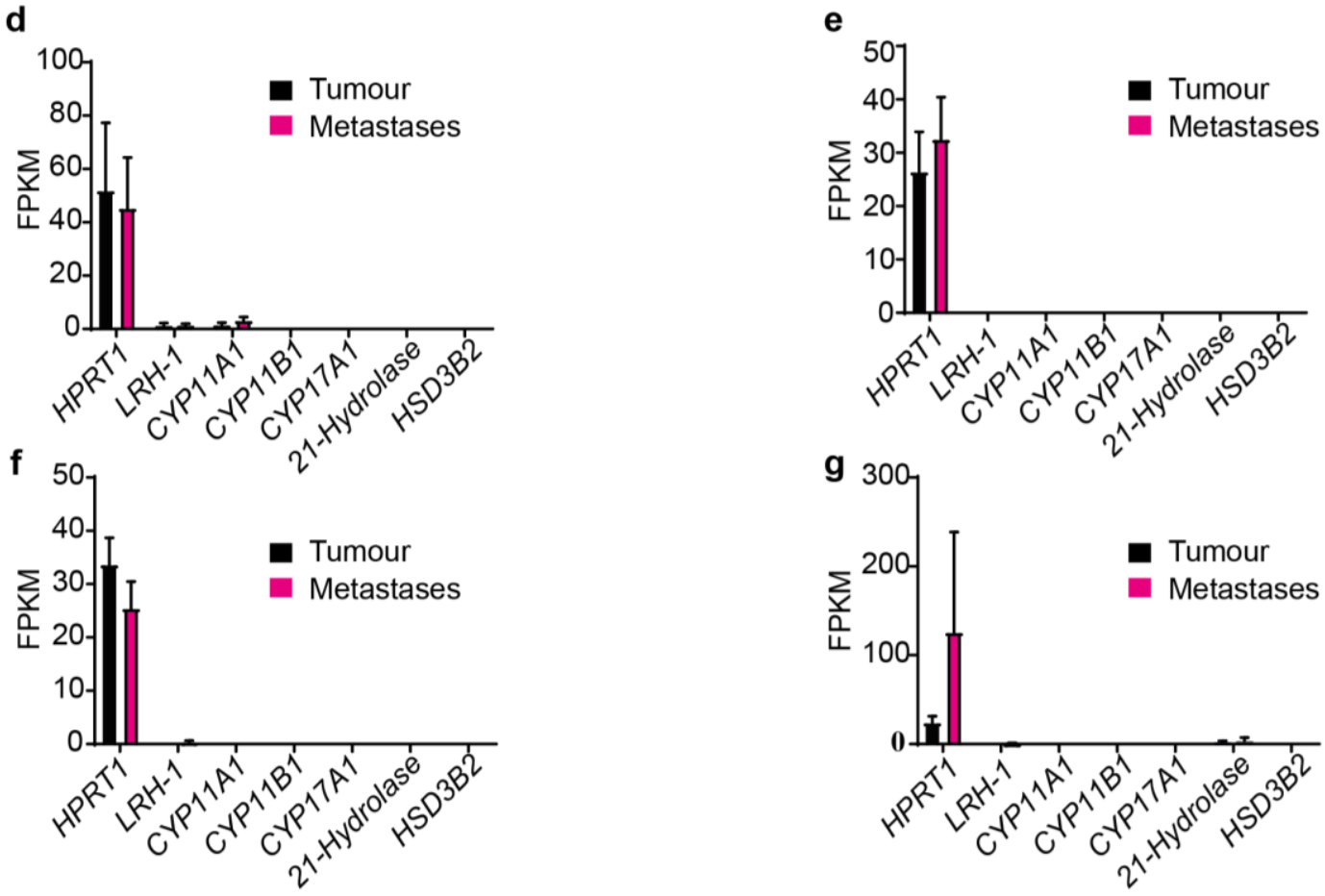

464 Extended Data Figure 2. GR activation in distant metastases and CTC. a, ISMARA transcription factor 465 activity plot of tumour, lung metastases, and CTC in MDA-MB 231 model, $n=3$ biological replicates. b, 466 ISMARA transcription factor analysis of PDX models, $n=3-4$ biological replicates. c, GR transcription 467 factor binding sites in the lung metastases of PDX1, 2 and 3, MDA-MB 231 and Balb-NeuT model ${ }^{16}$, $n=3$ 4684 biological replicates for PDX and MDA-MB 231 model. d-g, Expression of genes involved in 469 glucocorticoid synthesis and HPRT1 as an internal control d, MDA-MB 231; e, PDX1; f, PDX2 and g, 470 PDX3 model, mean \pm s.d, $n=3-4$ biological replicates. 
a
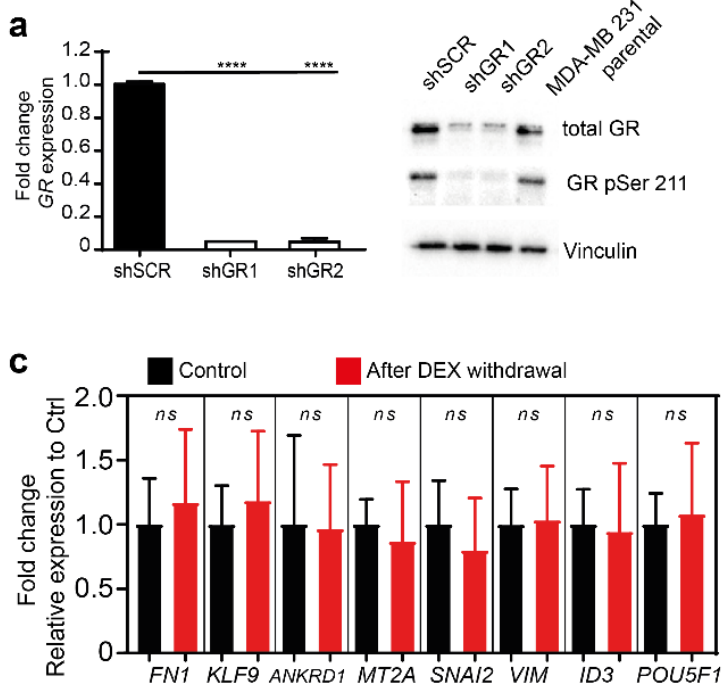

e

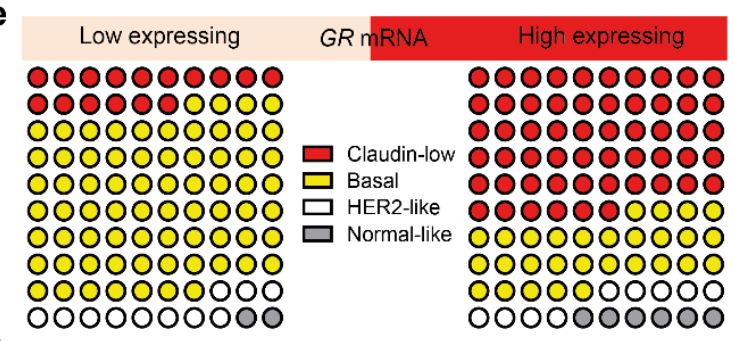

b

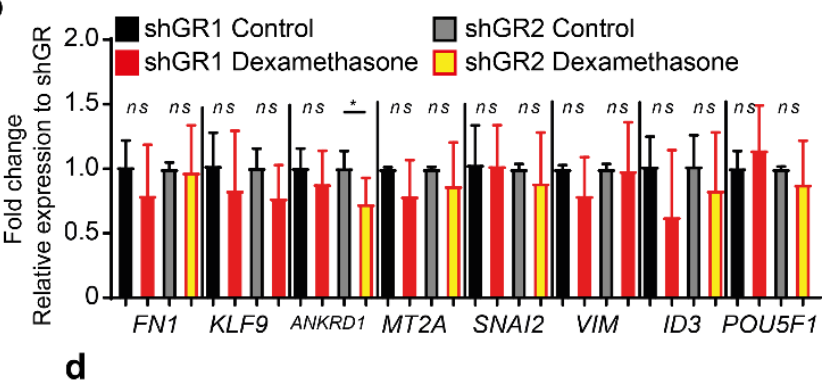

METABRIC, Nature 2012 \& Nat Commun 2016

\begin{tabular}{|l|l|r|r|}
\hline \multirow{4}{*}{ Gene } & $p$-value & \multicolumn{1}{c|}{ Log-odd ratio } \\
\cline { 2 - 4 } & KLF9 & $<0.001$ & 1.743 \\
\cline { 2 - 4 } & ID3 & $<0.001$ & 1.700 \\
\cline { 2 - 4 } GR & POU5F1 & 0.002 & 1.213 \\
\cline { 2 - 4 } & SNAI2 & 0.026 & 0.971 \\
\cline { 2 - 4 } & VIM & $<0.001$ & 2.221 \\
\cline { 2 - 4 } & FN1 & 0.074 & 0.777 \\
\cline { 2 - 4 } & ANKRD1 & 0.325 & -0.862 \\
\cline { 2 - 4 } & MT2A & 0.002 & 0.983 \\
\hline
\end{tabular}

f

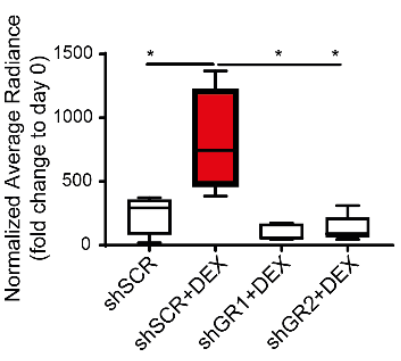

\begin{tabular}{l|cc} 
Control & \multicolumn{2}{|c}{ Dexamethasone } \\
\hline shSCR & shSCR & shGR1 \\
\hline & shGR2
\end{tabular}

\begin{tabular}{lllll}
2.0 & 1.5 & 1.0 & $0.5 \times 10^{9}$ & $\begin{array}{l}\text { Radiance } \\
\left(\mathrm{p} / \mathrm{sec} / \mathrm{cm}^{2} / \mathrm{sr}\right) \\
\mathrm{min}=3.0 \mathrm{e}) \\
\max =2.36 \mathrm{e} 9\end{array}$ \\
\hline
\end{tabular}

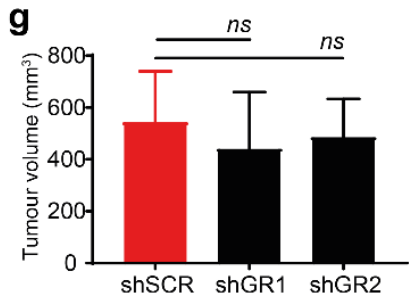

h

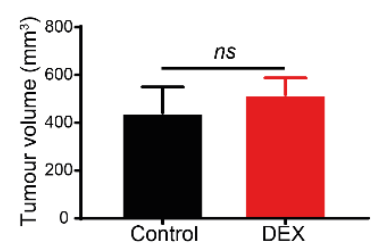

i

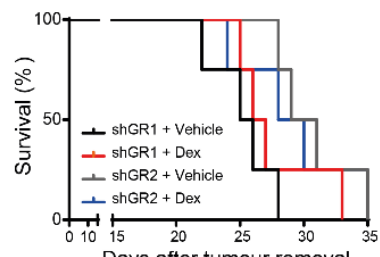

473 Extended Data Figure 3. Glucocorticoids escalate colonization via GR and expression of $G R$ signature

474 correlates with claudin-low breast cancer. a, GR down-regulation in MDA-MB 231 measured by qPCR

475 (left) and Western-blot (right). Mean \pm s.d., $n=3$ biological replicates, $* * * * P<0.0001$, two-tailed Student's

$476 t$-test. b, GR down-regulated MDA-MB 231 failed to express GR activation marker gene set upon treatment

477 with DEX, mean \pm s.d., $n=3$ biological replicates each measured in technical duplicates, $n s=$ non-significant, 
$478 * P<0.05$, two-tailed Student's $t$-test. $\mathbf{c}$, Expression of GR targets three weeks upon discontinuation of GR 479 activation by dexamethasone, mean \pm s.d., $n=3$ biological replicates each measured in technical duplicates, $480 n s=$ non-significant, two-tailed Student's $t$-test. d, Co-expression of GR activation gene set with GR, $481 n=2509$. e, Breast cancer expressing high GR mRNA were enriched in claudin-low profile, $n=299$. f, 482 Bioluminescence imaging of animals two weeks after i.v. injection of control and GR activated shGR or 483 control MDA-MB 231 cells. $n=5$ mice, $* P<0.05$, two-tailed Student's $t$-test. $\mathbf{g}$, GR down-regulation did not 484 impact tumour volume, mean \pm s.d., $n s=$ non-significant, $n=14$, Student's $t$-test. h, Tumour volumes initiated 485 of ex vivo GR activated and control MDA-MB 231 cells, $n=5$ mice, $n s=$ non-significant, two-tailed 486 Student's $t$-test. i, Kaplan-Meier survival analysis of mice injected with GR down-regulated MDA-MB 231 487 cells and treated with DEX or PBS, $n=5$ mice per group, Log-rank test. 
a

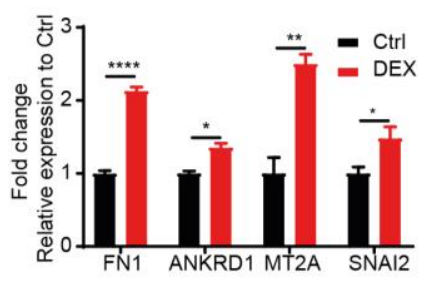

b

GO ID Biological process Count in gene set FDR GO.0030036 Actin cytoskeleton organization GO.0007010 Cytoskeleton organization

GO.0007169 Transmembrane receptor protein tyrosine kinase signaling pathway

GO.0006950 Response to stress

Go.0046496 Bicotinamide nucleotide metabolic process

GO.0006006 Glucose metabolic process

Go.0043666 Regulation of phosphoprotein phosphatase activity

Go.0051246 Regulation of protein metabolic process 790.000176

\section{d}

\begin{tabular}{|l|l|l|l|}
\hline Process & $P$-value & FDR & Ratio \\
\hline Development_EGFR signaling pathway & $1.293 \mathrm{e}-7$ & $3.361 \mathrm{e}-5$ & $8 / 71$ \\
\hline Signal transduction_ERK1/2 signaling pathway & $6.474 \mathrm{e}-6$ & $5.271 \mathrm{e}-4$ & $5 / 32$ \\
\hline Immune response_IL-33 signaling pathway & $8.726 \mathrm{e}-6$ & $5.672 \mathrm{e}-4$ & $6 / 58$ \\
\hline Immune response_IL-9 signaling pathway & $1.180 \mathrm{e}-5$ & $6.605 \mathrm{e}-4$ & $5 / 36$ \\
\hline Development_VEGF signaling via VEGFR2 - generic cascades & $1.270 \mathrm{e}-5$ & $6.605 \mathrm{e}-4$ & $7 / 93$ \\
\hline Development_HGF signaling pathway & $4.464 \mathrm{e}-5$ & $1.548 \mathrm{e}-3$ & $5 / 47$ \\
\hline Pathways of EMT in cancer cells & $6.658 \mathrm{e}-5$ & $1.902 \mathrm{e}-3$ & $5 / 51$ \\
\hline Cytoskeleton remodeling_FAK signaling & $1.140 \mathrm{e}-4$ & $2.578 \mathrm{e}-3$ & $5 / 57$ \\
\hline Development_Prolactin receptor signaling & $1.240 \mathrm{e}-4$ & $2.623 \mathrm{e}-3$ & $5 / 58$ \\
\hline Immune response_IL-6 signaling pathway via MEK/ERK and PI3K/AKT cascades & $3.921 \mathrm{e}-4$ & $4.634 \mathrm{e}-3$ & $5 / 74$ \\
\hline Development_G-CSF signaling & $7.565 \mathrm{e}-4$ & $7.868 \mathrm{e}-3$ & $4 / 49$ \\
\hline
\end{tabular}

C

$38 \quad 1.19 \mathrm{e}-12$

$49 \quad 2.54 \mathrm{e}-09$

$39 \quad 1.54 \mathrm{e}-06$

$107 \quad 1.53 \mathrm{e}-05$

$12 \quad 2.09 \mathrm{e}-05$

$15 \quad 3.87 e-05$

$\begin{array}{ll}9 & 0.000106\end{array}$

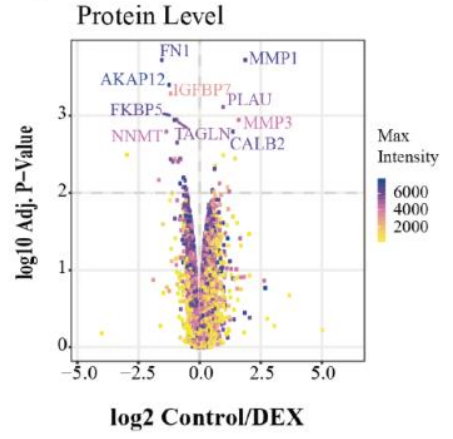

Extended Data Figure 4. Differential protein expression upon GR activation. a, Protein expression of

491 GR activation markers, mean \pm s.d., $* P<0.05, * * P<0.01, * * * * P<0.0001$, two-tailed Student's $t$-test. $\mathbf{b}$,

492 String proteome network analysis. Differentially regulated networks upon DEX treatment of MDA-MB

493231 cells. c, Volcano plot of differentially expressed proteins upon GR activation in MDA-MB 231, $n=3-4$

494 biological replicates, FDR $<0.05$. d, Metacore analysis of differentially activated networks measured by 495 phosphoproteomics. 
a

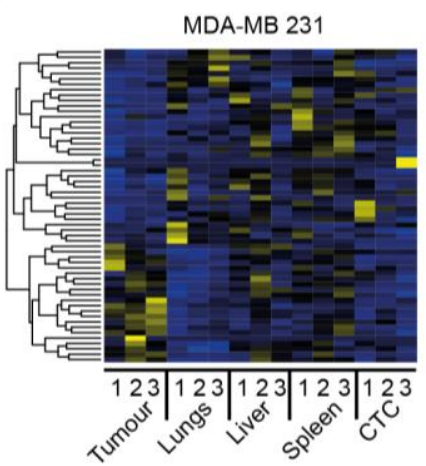

d

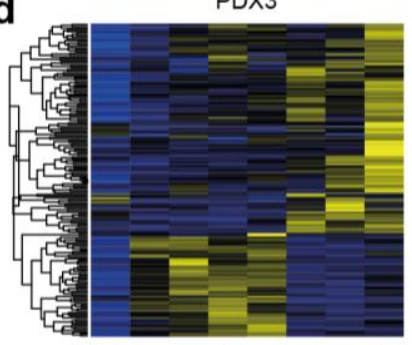

497

498

499

500

501

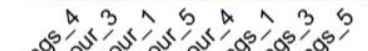

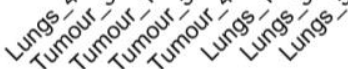

b

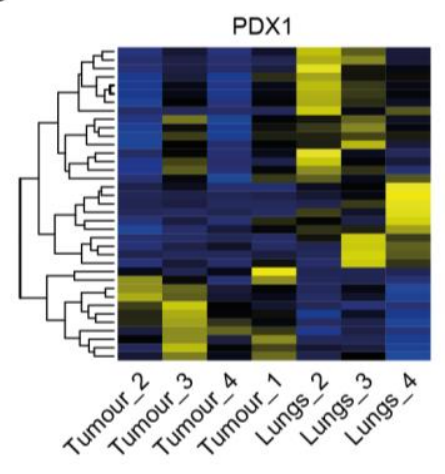

C

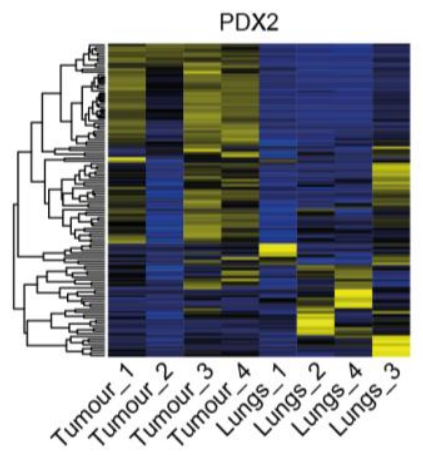

8 Extended Data Figure 5. Differential expression of protein kinases in tumours and matched 99 metastases. Expression of protein kinases in a, MDA-MB 231; b, PDX1; c, PDX2 and d, PDX3, n=3-4 00 biological replicates, $P<0.05$, fold change $\geq 2$. 
a

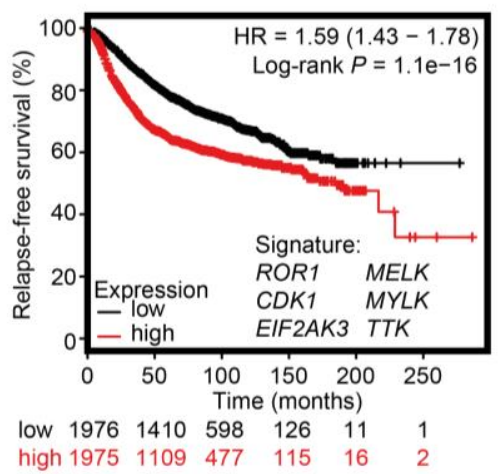

b

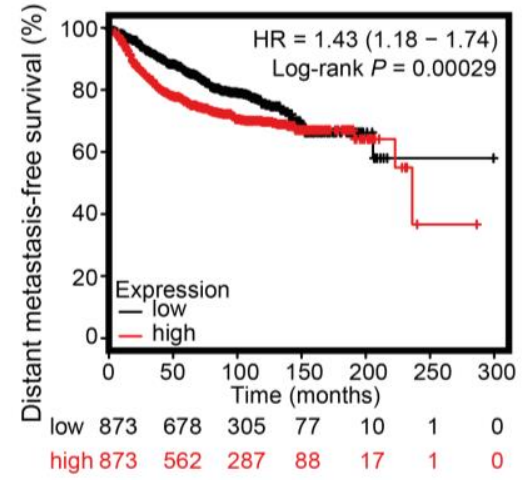

c

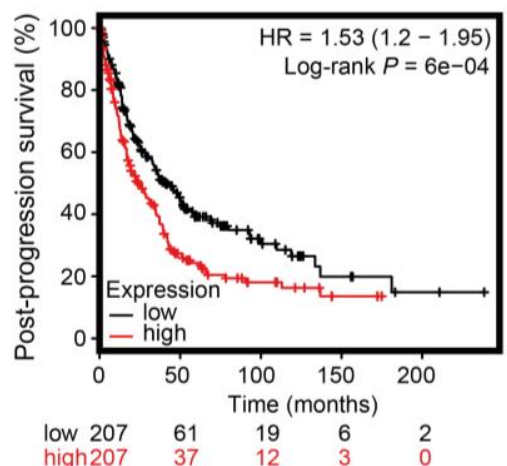

d
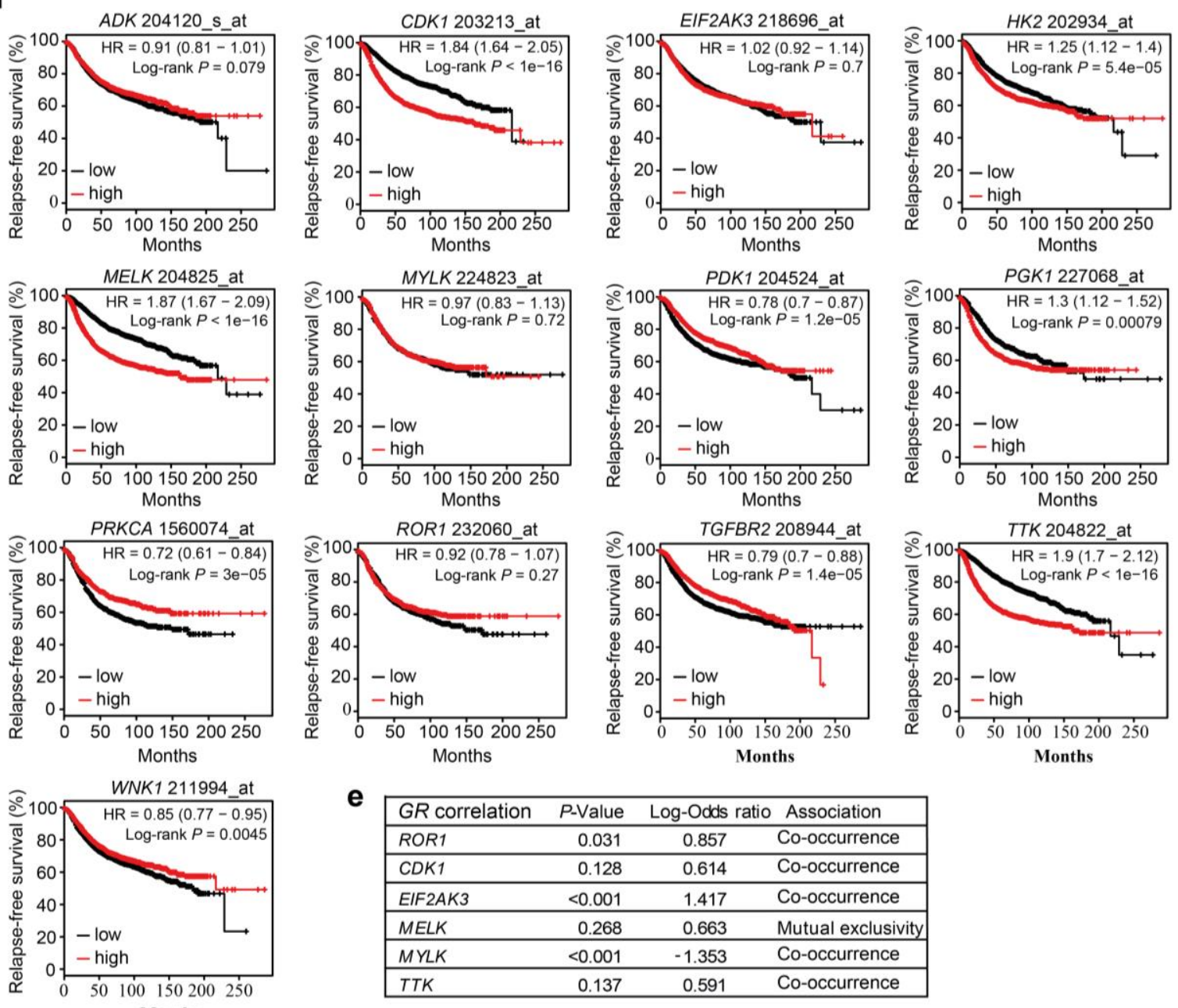

e

\begin{tabular}{|lrrl|}
\hline GR correlation & $P$-Value & Log-Odds ratio & Association \\
\hline ROR1 & 0.031 & 0.857 & Co-occurrence \\
\hline CDK1 & 0.128 & 0.614 & Co-occurrence \\
\hline EIF2AK3 & $<0.001$ & 1.417 & Co-occurrence \\
\hline MELK & 0.268 & 0.663 & Mutual exclusivity \\
\hline MYLK & $<0.001$ & -1.353 & Co-occurrence \\
\hline TTK & 0.137 & 0.591 & Co-occurrence \\
\hline
\end{tabular}

Months

503 Extended Data Figure 6. GR activation increases expression of kinases predictive of breast cancer

504 survival. Survival based on the expression of the protein kinase signature up-regulated in the metastases.

505 a, Relapse-free survival, $n=1764, P=7.7 \mathrm{e}-7$, Log-rank test; b, Distant metastases-free survival $n=664$, $P=0.00034$, Log-rank test; c, Post-progression survival, $n=173, P=0.084$, Log-rank test. d, Individual 
507 protein kinases, relapse-free survival, $n=1764$, Log-rank test. e, Co-occurrence of $G R$ and protein kinases 508 in breast cancer publically available datasets, $n=2509^{21,22}$.

509 

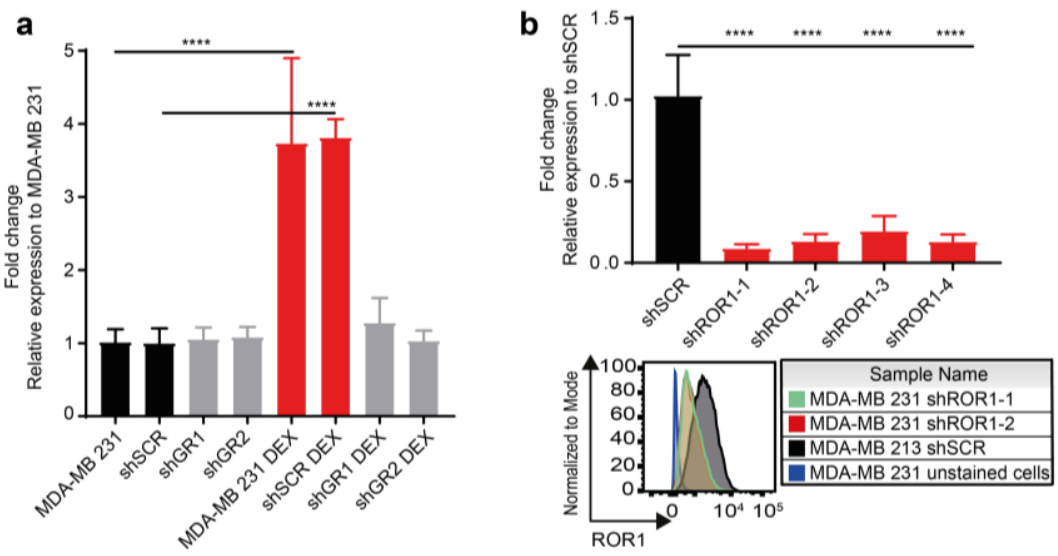

c
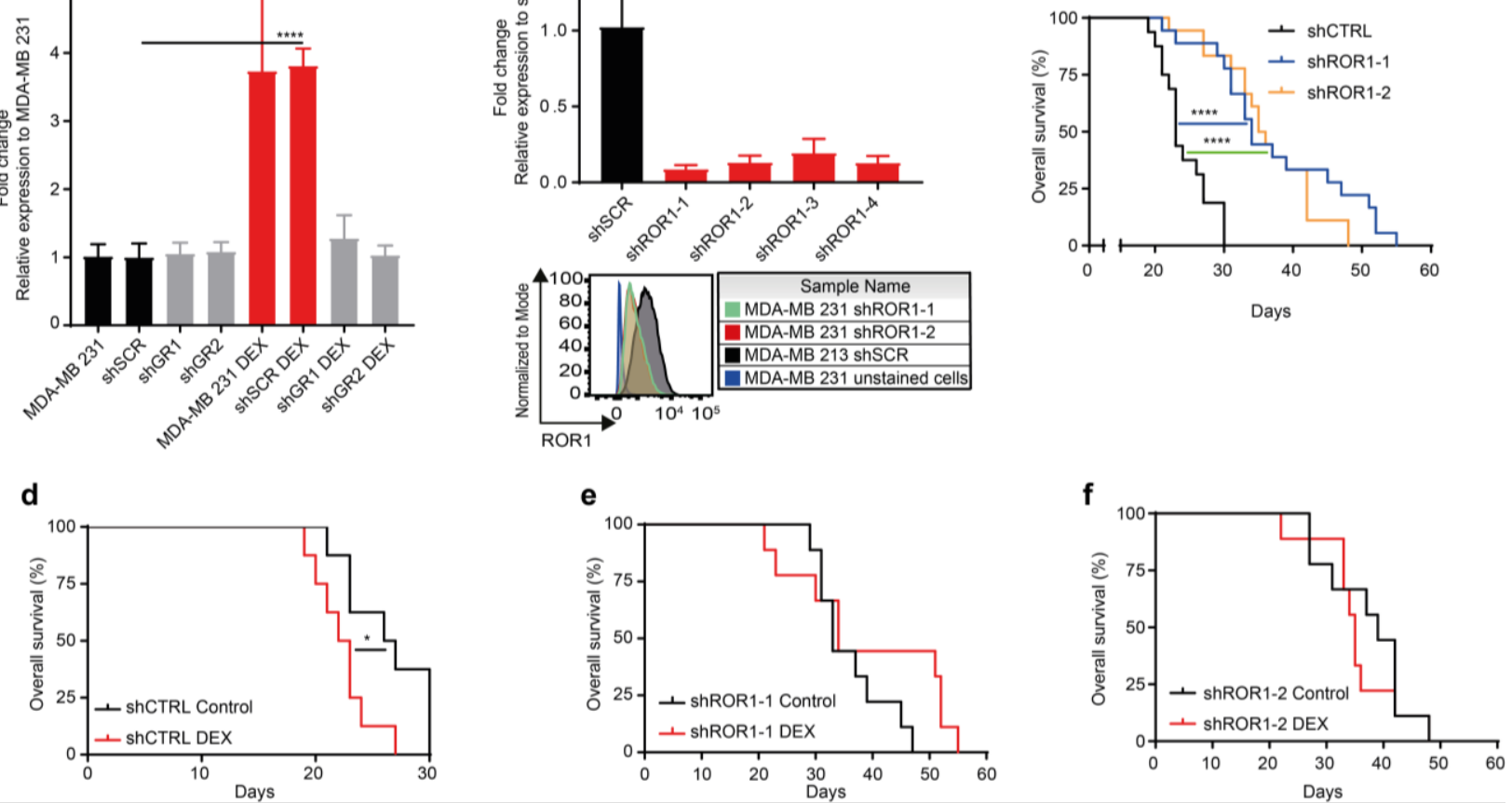

Extended Data Figure 7. ROR1 mediates GR induced lung metastatic colonization. a, ROR1

512 expression in control and GR down-regulated cells, mean \pm s.d., $n=3$ biological replicates, two-tailed

513 Student's $t$-test, $* * * * P<0.0001$. b, Down-regulation of ROR1 in MDA-MB 231 cells, mean \pm s.d., $n=3$

514 biological replicates, two-tailed Student's $t$-test, $* * * * P<0.0001$. Expression was measured by qPCR (up)

515 or flow cytometry (down). c, Kaplan-Meier post tumour resection survival analysis of mammary fat-pad

516 injected animals with control or ROR1 down-regulated MDA-MB 231 cells, $n=10$ mice biological

517 replicates, $* * * * P<0.0001$, Log-rank test. d, Kaplan-Meier post tumour resection survival analysis of

518 animals injected with MDA-MB 231 cells, $n=5$ mice per group, $* P<0.05$, Log-rank test. e, Kaplan-Meier

519 post tumour resection survival analysis of animals injected with GR down-regulated MDA-MB 231 cells

520 (shGR1), $n=5$ mice per group, $P=0.17$, Log-rank test. f, Kaplan-Meier post tumour resection survival

521 analysis of animals injected with GR down-regulated MDA-MB 231 cells (shGR1), $n=5$ mice per group,

$522 P=0.47$, Log-rank test. 
Figure 1. Increase of GR activation in distant metastases. a, Experimental design for the study of tumour heterogeneity in PDX and MDA-MB 231 model. MDA-MB 231 cells were injected in the mammary fatpad of female NSG mice. Cells isolated from the "tumour" (tumour cells found in the mammary fat-pad after orthotopic injection/transplantation) or matched "metastases" (cancer cells found in the distant sites such as lungs, liver etc. weeks after tumour resection) were sorted by FACS. b, Principal component analysis of tumours and matched metastases in MDA-MB 231 model clustered transcriptional profiles based on the site of metastases. The names in PCA plot represent tumour cells isolated from the indicated site, while the numbers within the names indicate a mouse; $n=3$ biological replicates. c, Heatmap of differentially expressed genes between tumours and lung metastases in MDA-MB 231 model; 280 genes yellow $=$ up-regulation, blue $=$ down-regulation, fold change $\geq 2, \operatorname{FDR}<0.05, n=3$ biological replicates. d, Ingenuity Pathway Analysis revealed GR and GR ligands as the Upstream Regulators of the lung metastatic phenotype, $n=3$ biological replicates. Increased levels of $\mathbf{e}$, cortisol, $\mathbf{f}$, corticosterone and $\mathbf{g}$, ACTH in the plasma of animals prior to tumour resection and animals with the distant metastases. Mean and single data points are represented, $n=8-12$ mice, biological replicates, two-tailed Student's $t$-test, ${ }^{*} P<0.05, * * P<0.01$.

Figure 2. GR activation escalates metastatic colonization and reduces survival. a, MDA-MB 231 cells were propagated in the presence of dexamethasone (DEX) for 7 days, scale bar $200 \mu \mathrm{m}$. b. Expression of GR targets after prolonged GR activation by DEX was assessed by qPCR. Data are mean \pm s.d., $n=6$ biological replicates each measured in 2 experimental replicates; two-tailed Student's $t$-test, $* * P<0.01$, $* * * P<0.001, * * * * P<0.0001$. c, Box plot- GR activation increased lung colonization upon i.v. injection in MDA-MB 231 model. Right, bioluminescence imaging two weeks after cell injection, $n=7$ mice and d, 4T1 model $n=9$ mice, ${ }^{*} P<0.01$, two-tailed Student's $t$-test. e-h, Administration of DEX after tumour removal in orthotopic models reduced survival in f, MDA-MB $231 n=12-18$ mice, pooled data from 3 independent experiments, $\mathbf{g}$, PDX1, $n=7-8$ mice; and $\mathbf{h}, 4 \mathrm{~T} 1$ model, $n=9$ mice, $* P<0.05$, *** $P<0.001$, **** $P<0.0001$, Log-rank test.

Figure 3. GR activation induces signaling networks and protein kinases implicated in breast cancer progression. a, Heatmap of differentially expressed proteins between MDA-MB 231 cells propagated in presence of DEX ( $n=3$ “control” biological replicates, $n=4$ "DEX” biological replicates, median expression, FDR $<0.01$; yellow $=$ up-regulation, blue $=$ down-regulation). $\mathbf{b}$, Differential peptide phosphorylation of GR activated and control MDA-MB 231 cells ( $n=3$ control biological replicates, $n=4$ DEX biological replicates, median expression, FDR $<0.05$ ). c, Overlap of differentially regulated protein kinases in the lung metastases of MDA-MB 231 model and in vitro propagated cells.

Figure 4. ROR1 mediates GR induced lung metastatic colonization. a, Relapse-free survival of patients with ROR1 signature (G-2-0 Kaplan-Meier), n=4029, Log-rank test. b, Flow cytometry of ROR1 
expression in tumour and matched metastases of MDA-MB 231 and PDX1, $n=3$ biological replicates. c, Kaplan-Meier survival analysis of animals inoculated i.v. with control or shROR1 MDA-MB 231 cells, $n=10$ mice per group, $* * * * P<0.0001$, Log-rank test. d, Experimental metastases assay (i.v.) and in vivo bioluminescence imaging of animals inoculated with GR activated or control MDA-MB 231 downregulated for ROR1 or shSCR, $* P<0.05, n s=$ non-significant, two-tailed Student's $t$-test. e-g, Kaplan-Meier survival analysis of animals inoculated i.v. with e, shSCR, f, shROR1-1 and g, shROR1-2 MDA-MB 231 cells propagated in presence of DEX or vehicle, $n=5$ mice per group, $* P<0.05$, Log-rank test.

\section{Extended Data Figure 1. Increase of glucocorticoid receptor activation in breast cancer metastases.}

a, Tumour and matched lungs, liver, ovary and spleen metastases in MDA-MB 231 model (hematoxylin and eosin staining), scale bar $200 \mu \mathrm{m}$. Right- frequency of metastases detected in the distant organs upon tumour resection, $n=10$ mice from independent experiments. b, FACS analysis of organs affected with distant metastases of MDA-MB 231 model, $n=10$ mice from independent experiments. c, Tumour growth kinetics after orthotopic transplantation in MDA-MB 231 and PDX models, $n=5$ biological replicates. Lower; Tumour and matched lung metastases in PDX1 and PDX2 models (hematoxylin and eosin staining), scale bar $200 \mu \mathrm{m}$. d, Tumour and matched lung, liver, ovary metastases in PDX3 model (hematoxylin and eosin staining), scale bar $200 \mu \mathrm{m}$. Right- Frequency of metastases detected in the distant organs upon tumour resection, $n=10$ mice from independent experiments. e, FACS analysis of tumour and organs with distant matched metastases in PDX3 model, $n=5$ biological replicates. f, Principal component analysis of tumours and matched metastases in PDX3, n=3-4 biological replicates. g, MDA-MB 231- Heatmap of differentially expressed genes between tumour and liver metastases; right- Upstream regulator analysis (Ingenuity Pathway Analysis- IPA), $n=3$ biological replicates, fold change $\geq 2$, FDR $<0.05$; $\mathbf{h}$, MDA-MB 231- Heatmap of differentially expressed genes between tumour and CTC; right- Upstream regulator analysis (Ingenuity Pathway Analysis- IPA), $n=3$ biological replicates, fold change $\geq 2$, FDR $<0.05$; i, MDAMB 231- Heatmap of differentially expressed genes between tumour and Spleen; right- Upstream regulator analysis (Ingenuity Pathway Analysis- IPA), $n=3$ biological replicates, fold change $\geq 2, \operatorname{FDR}<0.05 ; \mathbf{j}$, PDX1- Heatmap of differentially expressed genes between tumour and lung metastases, $n=3-4$ biological replicates, fold change $\geq 2$, FDR $<0.05$. $\mathbf{k}$, PDX2- Heatmap of differentially expressed genes between tumour and lung metastases, $n=4$ biological replicates, fold change $\geq 2$, FDR $<0.05$. I, PDX3- Heatmap of differentially expressed genes between tumour and lung metastases, $n=4$ biological replicates, fold change $\geq 2$, FDR $<0.05$. m, PDX3- Heatmap of differentially expressed genes between tumour and liver metastases, $n=4$ biological replicates, fold change $\geq 2$, FDR $<0.05$.

Extended Data Figure 2. GR activation in distant metastases and CTC. a, ISMARA transcription factor activity plot of tumour, lung metastases, and CTC in MDA-MB 231 model, $n=3$ biological replicates. b, 
ISMARA transcription factor analysis of PDX models, $n=3-4$ biological replicates. c, GR transcription factor binding sites in the lung metastases of PDX1, 2 and 3, MDA-MB 231 and Balb-NeuT model ${ }^{16}, n=3$ 4 biological replicates for PDX and MDA-MB 231 model. d-g, Expression of genes involved in glucocorticoid synthesis and HPRT1 as an internal control d, MDA-MB 231; e, PDX1; f, PDX2 and g, PDX3 model, mean \pm s.d, $n=3-4$ biological replicates.

Extended Data Figure 3. Glucocorticoids escalate colonization via GR and expression of $G R$ signature correlates with claudin-low breast cancer. a, GR down-regulation in MDA-MB 231 measured by qPCR (left) and Western-blot (right). Mean \pm s.d., $n=3$ biological replicates, $* * * * P<0.0001$, two-tailed Student's $t$-test. b, GR down-regulated MDA-MB 231 failed to express GR activation marker gene set upon treatment with DEX, mean \pm s.d., $n=3$ biological replicates each measured in technical duplicates, $n s=$ non-significant, $* P<0.05$, two-tailed Student's $t$-test. c, Expression of GR targets three weeks upon discontinuation of GR activation by dexamethasone, mean \pm s.d., $n=3$ biological replicates each measured in technical duplicates, $n s=$ non-significant, two-tailed Student's $t$-test. d, Co-expression of GR activation gene set with GR, $n=2509$. e, Breast cancer expressing high GR mRNA were enriched in claudin-low profile, $n=299$. f, Bioluminescence imaging of animals two weeks after i.v. injection of control and GR activated shGR or control MDA-MB 231 cells. $n=5$ mice, $* P<0.05$, two-tailed Student's $t$-test. g, GR down-regulation did not impact tumour volume, mean \pm s.d., $n s=$ non-significant, $n=14$, Student's $t$-test. $\mathbf{h}$, Tumour volumes initiated of ex vivo GR activated and control MDA-MB 231 cells, $n=5$ mice, $n s=$ non-significant, two-tailed Student's $t$-test. i, Kaplan-Meier survival analysis of mice injected with GR down-regulated MDA-MB 231 cells and treated with DEX or PBS, $n=5$ mice per group, Log-rank test.

Extended Data Figure 4. Differential protein expression upon GR activation. a, Protein expression of GR activation markers, mean \pm s.d., ${ }^{*} P<0.05$, $* * P<0.01$, $* * * * P<0.0001$, two-tailed Student's $t$-test. $\mathbf{b}$, String proteome network analysis. Differentially regulated networks upon DEX treatment of MDA-MB 231 cells. c, Volcano plot of differentially expressed proteins upon GR activation in MDA-MB 231, $n=3-4$ biological replicates, FDR $<0.05$. d, Metacore analysis of differentially activated networks measured by phosphoproteomics.

Extended Data Figure 5. Differential expression of protein kinases in tumours and matched metastases. Expression of protein kinases in a, MDA-MB 231; b, PDX1; c, PDX2 and d, PDX3, n=3-4 biological replicates, $P<0.05$, fold change $\geq 2$.

Extended Data Figure 6. GR activation increases expression of kinases predictive of breast cancer survival. Survival based on the expression of the protein kinase signature up-regulated in the metastases. a, Relapse-free survival, $n=1764, P=7.7 \mathrm{e}-7$, Log-rank test; $\mathbf{b}$, Distant metastases-free survival $n=664$, 
$P=0.00034$, Log-rank test; c, Post-progression survival, $n=173, P=0.084$, Log-rank test. d, Individual protein kinases, relapse-free survival, $n=1764$, Log-rank test. e, Co-occurrence of $G R$ and protein kinases in breast cancer publically available datasets, $n=2509^{21,22}$.

Extended Data Figure 7. ROR1 mediates GR induced lung metastatic colonization. a, ROR1 expression in control and GR down-regulated cells, mean \pm s.d., $n=3$ biological replicates, two-tailed Student's $t$-test, $* * * * P<0.0001$. b, Down-regulation of ROR1 in MDA-MB 231 cells, mean \pm s.d., $n=3$ biological replicates, two-tailed Student's $t$-test, $* * * * P<0.0001$. Expression was measured by qPCR (up) or flow cytometry (down). c, Kaplan-Meier post tumour resection survival analysis of mammary fat-pad injected animals with control or ROR1 down-regulated MDA-MB 231 cells, $n=10$ mice biological replicates, $* * * * P<0.0001$, Log-rank test. d, Kaplan-Meier post tumour resection survival analysis of animals injected with MDA-MB 231 cells, $n=5$ mice per group, ${ }^{*} P<0.05$, Log-rank test. e, Kaplan-Meier post tumour resection survival analysis of animals injected with GR down-regulated MDA-MB 231 cells (shGR1), $n=5$ mice per group, $P=0.17$, Log-rank test. f, Kaplan-Meier post tumour resection survival analysis of animals injected with GR down-regulated MDA-MB 231 cells (shGR1), $n=5$ mice per group, $P=0.47$, Log-rank test. 